\title{
QUASARS IN THE COSMOS FIELD
}

\author{
M. K. M. Prescott, ${ }^{1}$ C. D. Impey, ${ }^{1}$ R. J. Cool, ${ }^{1}$ and N. Z. Scoville $^{2}$ \\ Received 2005 December 19; accepted 2006 February 10
}

\begin{abstract}
We obtained medium-resolution spectra of 336 quasar candidates in the COSMOS HST Treasury field using the MMT $6.5 \mathrm{~m}$ telescope and the Hectospec multiobject spectrograph. Candidates were drawn from the Sloan Digital Sky Survey (SDSS) DR1 catalog using quasar flags set by the SDSS multicolor quasar target selection algorithm. In this paper we present our discovery spectra from $1.39 \mathrm{deg}^{2}(69.5 \%$ of the COSMOS field $)$ and a discussion of the selection method and yields. We confirmed 95 quasars, including at least two BAL quasars; 80 of these are new quasars that do not appear in previous quasar confirmation follow-up studies. The candidates additionally included 184 compact emission-line galaxies, a third of which are likely type 2 AGNs, and 12 stars. The quasars span a range in magnitude of $18.3<g<22.5$ and a range in redshift of $0.2<z<2.3$. Our results are consistent with a lower limit quasar surface density from SDSS color selection of $102 \mathrm{deg}^{-2}$ down to $g=22.5$ over the entire COSMOS field. This work is the first step toward the eventual goal of setting up a grid of quasar absorption line probes of the $2 \mathrm{deg}^{2}$ field and of conducting a complete census of supermassive black holes in this well-studied survey region. The total quasar count at the conclusion of this study is 139 , making COSMOS one of the most densely sampled regions of sky where a grid of quasar sight lines can be used to probe the intervening volume.
\end{abstract}

Subject headings: quasars: emission lines — quasars: general — surveys

Online material: machine-readable table, extended figure set

\section{INTRODUCTION}

Quasar absorption-line studies of the intergalactic medium have uncovered a complex "web" of cosmic structure. Optimum studies of the cosmic web use a grid of distant quasars as a set of probes of the intervening matter. Absorbing material along the line of sight is identified in the quasar spectrum with an equivalent width and redshift and can be used to study the distribution, chemical enrichment, and clustering relative to luminous matter in the same volume. The Ly $\alpha$ forest absorption traces highly ionized hydrogen of low column density and low chemical enrichment (Meylan 1995). These absorbers are weakly clustered along the line of sight and are more closely associated with voids and large-scale structures than they are with individual bright galaxies (Grogin \& Geller 1998; Bahcall et al. 1993; Weymann et al. 1998; Penton et al. 2002). Metal absorption lines such as the $\mathrm{Mg}$ II $\lambda \lambda 2796,2800$ and $\mathrm{C}$ IV $\lambda \lambda 1548,1550$ doublets offer a complementary approach to the study of large-scale structure, as they trace massive galaxy halos via the cross section to metalenriched gas.

Unlike the targets of luminous matter surveys, quasar absorbers can be selected in ways that are largely unaffected by the cosmological $(1+z)^{-4}$ surface brightness dimming, surface brightness and morphology selection effects, $k$-corrections, and Malmquist bias. Thus, the advantage of absorbers as probes of the intergalactic medium is that they can be detected with an efficiency that does not depend strongly on redshift, given a sufficiently bright background quasar. On the other hand, faint quasar surveys are required to set up a dense enough grid of probes in a contiguous area to sample the full range of cosmic structures. Obtaining a complete picture of the large-scale structure involves

\footnotetext{
1 Steward Observatory, 933 North Cherry Avenue, University of Arizona, Tucson, AZ 85721; mprescott@as.arizona.edu, cimpey@as.arizona.edu,rcool@ as.arizona.edu.

2 Robinson Laboratory, California Institute of Technology, Pasadena, CA 91125; nzs@phobos.caltech.edu.
}

unifying these disparate studies of dim matter in the intergalactic medium (IGM) and luminous matter in galaxies.

The Cosmic Evolution Survey (COSMOS) is the largest HST (Hubble Space Telescope) survey ever undertaken with the ACS (Advanced Camera for Surveys) instrument (Scoville et al. 2006). The field is $1.4 \times 1.4$ square, aligned east-west, north-south, centered at $10^{\mathrm{h}} 00^{\mathrm{m}} 28^{\mathrm{s}} \mathrm{s},+02^{\circ} 12^{\prime} 21^{\prime \prime} 0$ (J2000.0), and accessible to all major observatories. Extensive multiwavelength observations of the field are in progress, including deep radio (Very Large Array [VLA]), UV (Galaxy Evolution Explorer [GALEX]), X-ray (XMM-Newton), and infrared (Spitzer) mapping and a densely sampled galaxy redshift survey using the VLT (Very Large Telescope). A total of 45,000 redshifts will be obtained within the $2 \mathrm{deg}^{2}$ region. The field size was chosen to span the largest known cosmic structures at $1<z<2$, and the volume encompassed by the galaxy redshift survey rivals that of the Sloan Digital Sky Survey (SDSS).

Building on the extensive spatial, wavelength, and redshift coverage contained in the COSMOS field, the eventual goal of this project is to build a large grid of quasars to yield threedimensional "tomography" of the IGM via Ly $\alpha$ absorption as well as three-dimensional measures of the relationship between galaxy halos selected by $\mathrm{Mg}$ II and $\mathrm{C}$ IV absorption and the visible baryons in luminous galaxies. Similar observations of individual quasar sight lines have been used to this effect to show evidence of strong overdensities (Heisler et al. 1989) and that $\mathrm{C}$ IV and $\mathrm{Mg}$ II have correlation power on scales up to $\sim 140 h_{70}^{-1} \mathrm{Mpc}$ (Loh et al. 2001). Absorber studies with multiple sight lines have been used to map out large-scale three-dimensional structures: Dinshaw \& Impey (1996) found a flattened structure at $z \sim 2$ that is $\sim 40 h_{70}^{-1}$ Mpc by $110 h_{70}^{-1} \mathrm{Mpc}$, while Williger et al. (1996) discovered a $20-50 h_{70}^{-1} \mathrm{Mpc}$ structure toward the south Galactic pole. Cohen et al. $(1996,1999)$ found that roughly half of the bright galaxies in deep galaxy redshift surveys are located in high-contrast structures separated by $70-430 h_{70}^{-1} \mathrm{Mpc}$ along the line of sight. Multiple sight lines can also be used to measure three-dimensional absorber clustering (Sargent \& Steidel 1987); 
Ly $\alpha$ absorbers seem to be weakly clustered on scales of 30 $40 h_{70}^{-1} \mathrm{Mpc}$ (Williger et al. 2000; Liske et al. 2000). The distribution of diffuse IGM baryons traced by Ly $\alpha$ and metal-line absorbers can be compared to the distribution of dark matter traced by weak lensing and the large-scale structure defined by galaxy redshift surveys.

This project must be approached in several stages. The first phase is the confirmation of quasars within the COSMOS field. Color-selection methods and existing surveys provide the candidate population, and low-resolution spectra are sufficient for identification of the broad quasar emission features (e.g., Ly $\alpha$, $\mathrm{C}$ IV, $\mathrm{C}$ III, and $\mathrm{Mg}$ II). The second phase involves high-resolution spectroscopic follow-up of confirmed quasars providing equivalent widths and identifications of absorbers along the line of sight toward the quasar. In the third and final phase, these absorber data, tracing the diffuse IGM and galaxy halos, will be merged with the COSMOS dark matter and galaxy redshift surveys in order to map out the three-dimensional distribution of dim and luminous matter. There is now the prospect of using COS (Cosmic Origins Spectrograph) on the HST to map Ly $\alpha$ at $0<$ $z<1.6$, below the atmospheric cutoff, and of comparing directly to galaxy redshifts measured with the VLT.

The goal of the current work is to begin setting up a grid of quasars across the COSMOS field using preexisting quasar samples and selection methods. This paper presents the results of our first quasar confirmation run using a large sample of previously untargeted quasar candidates selected from the SDSS DR1 (Data Release 1) photometric catalog (York et al. 2000; Abazajian et al. 2003). More recent work within the extended COSMOS collaboration is yielding larger and fainter quasar samples (Impey et al. 2006; Trump et al. 2006). In $\S 2$ we discuss our sample selection, and in $\S 3$, our observations and reduction procedures. Sections 4 and 5 present our results and analysis, and we review our conclusions in $\S 6$. Discovery spectra are shown in the Appendix.

\section{SAMPLE SELECTION}

We derived our target sample from the SDSS DR1 photometric catalog. As part of the SDSS quasar target selection, objects contained in the SDSS photometric catalogs were assigned a number of flags related to their likelihood of being a quasar and whether or not they were to be targeted for follow-up SDSS spectroscopy. These determinations were based on finding outliers from the stellar locus in four-color space and matching SDSS sources against the FIRST (Faint Images of the Radio Sky at Twenty $\mathrm{cm}$ ) catalog of radio sources. The relevant flags are described by Richards et al. (2002b) and references therein. Vanden Berk et al. (2005) find empirically that the completeness of the SDSS selection algorithm is $94.9 \%$ down to the limiting magnitude of $i=19.1$, while that of the whole SDSS quasar survey is $89 \%$ due to image defects, unidentifiable spectra, and extended sources. Objects selected as quasar candidates that lie outside the SDSS spectroscopic magnitude limits of $15<i^{*}<$ 19.1 for low-redshift and $15<i^{*}<20.2$ for high-redshift selection are flagged accordingly and left to follow-up studies such as the current work.

Using the SDSS quasar flags, we compiled a list of quasar candidates, removing the 48 objects that had previously been targeted by SDSS (as of DR1), resulting in 1391 targets over the entire COSMOS field. We then made two cuts, keeping only those targets with $u$-band rms errors $<0.3 \mathrm{mag}$ and $g<22.5$. Since photometric errors are highest in the $u$ band, and since ultraviolet excess is a key discriminant of a quasar, we made the $u$-band error cut in order to remove targets with unreliable pho-

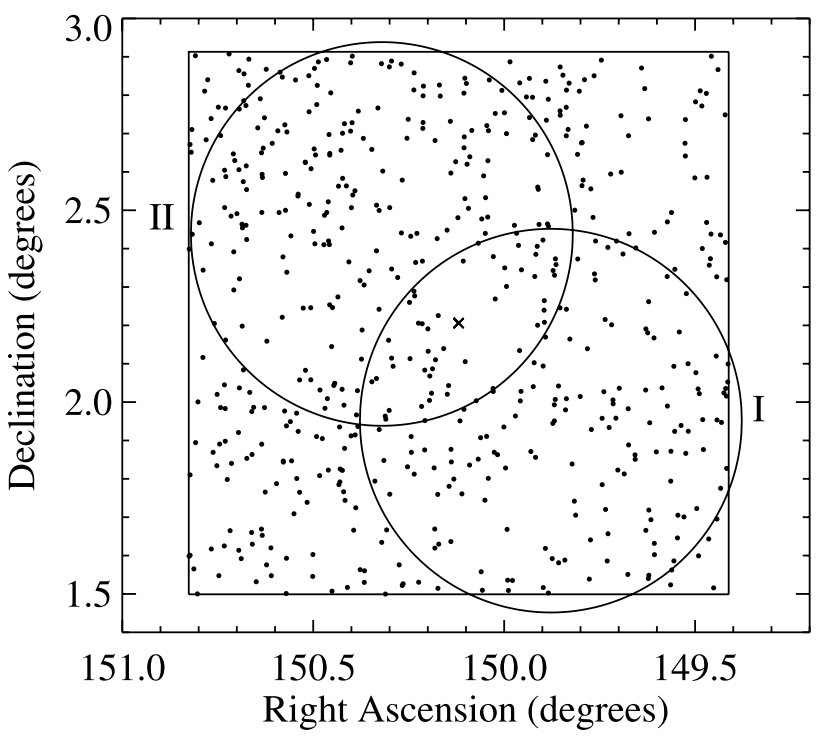

FIG. 1.-Map of the spatial distribution of 566 SDSS quasar candidates within the $2 \mathrm{deg}^{2}$ COSMOS field, overlaid with the two $1^{\circ}$ diameter Hectospec pointings used during the observations. The COSMOS field is the inscribed square, with its center indicated as a cross at $10^{\mathrm{h}} 00^{\mathrm{m}} 28^{\mathrm{s}} \mathrm{s},+02^{\circ} 12^{\prime} 21^{\prime \prime} 0(\mathrm{~J} 2000.0)$.

tometry. The $g$-magnitude limit was chosen as the faintest magnitude bin in which the candidates were well defined in a $u-g$ versus $g-r$ plot relative to typical stellar colors. After applying these cuts, we were left with 566 quasar candidate targets.

Figure 1 shows a map of the 566 target positions within the COSMOS field; a plot of $g$ magnitude versus $u-g$ color for the final target sample is given in Figure 2. The 43 quasars confirmed within the COSMOS field from SDSS DR1 and the additional 21 (of which 20 overlap our sample) confirmed up to and including the SDSS DR4 spectroscopic follow-up are shown in open circles (Adelman-McCarthy et al. 2006). Compared to the candidate sample for the present study, they are confined to magnitudes brighter than $g=20.5$ and slightly bluer $u-g$ colors.

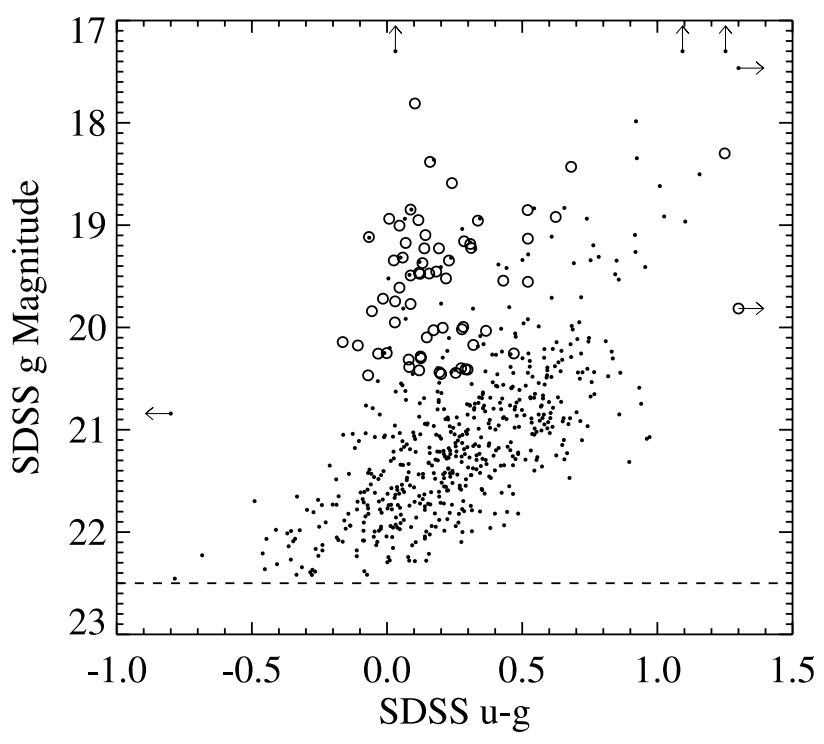

FIG. 2.-Color-magnitude plot of the SDSS photometrically selected targets (566 filled circles). The 64 quasars confirmed up to and including SDSS DR4 are shown as open circles. Improvements in photometry between SDSS DR1 and DR4 account for the slight offsets visible in a few cases. The dotted line indicates the $g<22.5$ cut applied. 


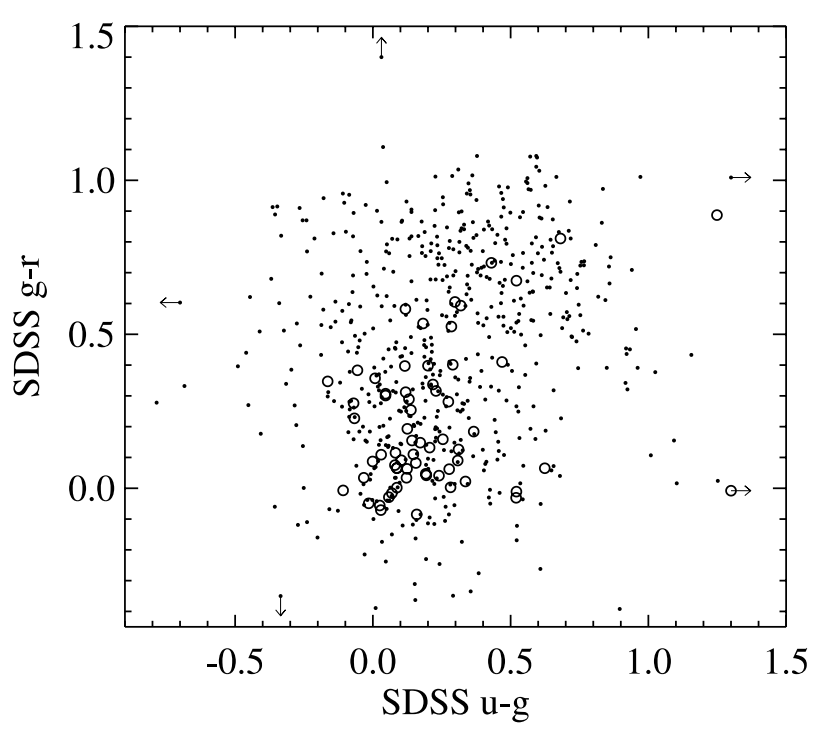

FIG. 3.-Color-color plot of SDSS colors for quasar candidates in this study (566 filled circles) and previously confirmed SDSS quasars (64 open circles). Improvements in photometry between SDSS DR1 and DR4 account for the slight offsets that appear in a few cases.

Similarly, Figure 3 shows the SDSS $g-r$ versus $u-g$ plot for our target sample and the previously confirmed SDSS quasars. The two populations cover similar regions of color-color space, with our target sample extending to redder $g-r$ colors. From this plot it is clear that our sample is not missing regions of colorcolor space with respect to the previously known and brighter SDSS quasar sample.

Magnitudes quoted in this study are observed SDSS magnitudes, uncorrected for Galactic extinction. For reference, the mean Galactic extinction for this field is $A_{g} \sim 0.07$ from Schlegel et al. (1998). The typical photometric error for SDSS $g$ and $r$ magnitudes is $\sim 0.25$.

\section{OBSERVATIONS AND REDUCTIONS}

\subsection{Observational Details}

Observations were carried out during the night of 2004 April 18 using the MMT $6.5 \mathrm{~m}$ telescope and the Hectospec multiobject spectrograph (Fabricant et al. 2004). Hectospec has 300 optical fibers, each 1".5 in diameter, which are positioned by two fiber robots to an accuracy of $\sim 0$.'2 in $\sim 5$ minutes. The resolution of the grating selected for this Hectospec setup was $6 \AA$. The minimum fiber spacing is roughly $20^{\prime \prime}$, but the actual fiber constraints for a particular observational setup are determined by the fiber positioning software. The peak final throughput for Hectospec, including the telescope optics, is $21 \%$ at $5000 \AA$. The Hectospec detector array is composed of two $2048 \times 4608$ pixel CCDs, with the gap positioned parallel to the dispersed spectrum. The grating used had 270 lines $\mathrm{mm}^{-1}$ and was blazed at $5200 \AA$. The full spectral coverage was $5800 \AA$, spanning from $\sim 3100$ to $9000 \AA$, at a dispersion of $1.21 \AA$ pixel $^{-1}$. Normal calibration frames were taken (dome flat, comparison arc lamp, and bias frames); no flux standards were necessary for this initial quasar confirmation phase.

The targets were divided between two overlapping pointings, as shown in Figure 1, which were observed for 60 and 80 minutes, respectively. Of the 566 SDSS quasar candidates in the COSMOS field, 388 were within the two pointings, and we obtained spectra of 336. The number of targets and sky fibers per pointing, pointing positions, individual integration times, and air
TABLE 1

Spectroscopic Observations

\begin{tabular}{|c|c|c|}
\hline Parameter & Pointing I & Pointing II \\
\hline 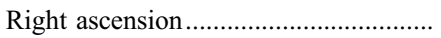 & 95930.65 & 100116.88 \\
\hline 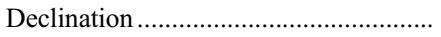 & +15705.94 & +2 2617.83 \\
\hline 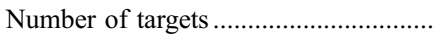 & 153 & 183 \\
\hline Number of sky fibers ............................... & 98 & 95 \\
\hline 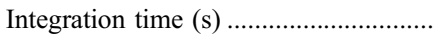 & 3600 & 4800 \\
\hline 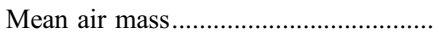 & 1.16 & 1.28 \\
\hline
\end{tabular}

NoTE.- Units of right ascension are hours, minutes, and seconds, and units of declination are degrees, arcminutes, and arcseconds.

masses are given in Table 1. As the field was setting during our observations, we used a longer integration time on the second pointing to partially compensate for the increase in air mass; the fractional quasar yields for the two pointings are similar: 0.27 for pointing I and 0.28 for pointing II.

Conditions during the observations were mostly clear, but image quality was affected by high winds. Two problems with the data were noticed after the run: an LED leak affecting the red end of the spectra ( $\sim 8000-9000 \AA)$ and a problem with the atmospheric dispersion corrector (ADC) causing lowered counts, particularly in the blue spectral region $(\sim 3000-4000 \AA)$. Fortunately, these issues presented only minor problems and did not prevent us from achieving the goals of this phase of the project.

\subsection{Data Reduction}

We reduced the Hectospec data with HSRED, ${ }^{3}$ an IDL package developed for reduction of data from the Hectospec and Hectochelle instruments on the MMT. The code draws heavily on the reduction pipeline used by SDSS. The 300 fiber trace locations were determined using dome flat spectra. These dome flat observations were further used to correct for the high-frequency flat-field variations in the observations and the CCD fringing. Sky subtraction was performed using dedicated sky fibers distributed throughout each field. The bright sky lines in each spectrum were used to refine the wavelength solution determined from a set of HeNeAr comparison spectra obtained at the beginning of the night.

Object classifications and redshifts were determined using a modified version of the SDSS redshift pipeline available publicly in the IDLSPEC2D package. Each spectrum was compared to a grid of galaxy, quasar, and star spectra. A $\chi^{2}$ minimization method was used to determine the best-fit spectral classification and redshift.

Verification by eye confirmed all but a handful of cases in which a low signal-to-noise ratio, target faintness, or the spectral problems described in $\S 3.1$ caused the cross-correlation method to fail. For these objects we measured the redshifts by hand using a flux-weighted mean and a simple Monte Carlo error estimation method. All objects were assigned a classification of QUASAR, QUASAR1, QUASAR?, QUASAR BAL, GALAXY, GALAXY1, GALAXY?, STAR, STAR?, or UNKNOWN. Table 2 gives a description of the observational criteria for each of these categories. Objects with secure classifications are all included in the final numbers for the overall categories of QUASAR, GALAXY, and STAR, while those with question marks and those in the UNKNOWN category remain without a secure identification and are not used in any subsequent statistical analysis.

\footnotetext{
${ }^{3}$ See http://mizar.as.arizona.edu/rcool/hsred/index.html.
} 
TABLE 2

Spectroscopic Classification

\begin{tabular}{|c|c|c|c|}
\hline Classification & Description & Inclusion in Final Count & Number \\
\hline 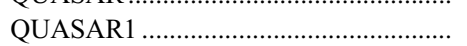 & Definite quasar with 1 broad line and additional piece of evidence ${ }^{b}$ & QUASAR & 24 \\
\hline 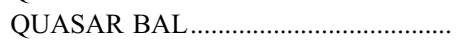 & Broad absorption line quasar & QUASAR & 2 \\
\hline GALAXY & Definite galaxy with 2 or more emission or absorption lines ${ }^{c}$ & GALAXY & 172 \\
\hline 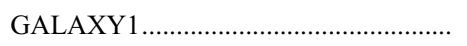 & Definite galaxy with 1 narrow line and additional piece of evidence ${ }^{\mathrm{d}}$ & GALAXY & 12 \\
\hline 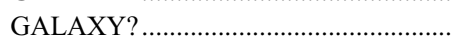 & Uncertain, probably a galaxy & $\ldots$ & 17 \\
\hline 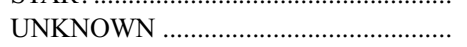 & No classification was possible & $\cdots$ & 7 \\
\hline
\end{tabular}

${ }^{\text {a }}$ Quasar broad emission line features, e.g., Ly $\alpha, \mathrm{C}$ IV, C III, Mg II, and $\mathrm{H} \alpha$.

${ }^{\mathrm{b}}$ More uncertain, but corroborating broad emission lines or spectral breaks outside the range 4000-8000 $\AA$.

c The 4000 Å break, $\mathrm{Ca}$ II $\mathrm{H}$ and $\mathrm{K}$ lines, galaxy emission lines, e.g., [O II], [O III] doublet.

${ }^{\mathrm{d}}$ More uncertain, but corroborating galaxy spectral features outside the range 4000-8000 $\AA$.

In many cases the LED leak and ADC error had an almost purely cosmetic effect on the data. However, for targets with redshifts between 0.3 and 0.5 , the $\mathrm{Mg}$ II emission line lies in the compromised blue end of the spectrum and $\mathrm{H} \alpha$ is located in the compromised red end, leaving only $\mathrm{H} \beta$ and the $[\mathrm{O}$ III] doublet lines for determining a redshift. In these cases, the combined loss of reliable spectral coverage meant that a few additional objects could not be confidently identified or have their redshifts measured.

\section{RESULTS}

From these MMT observations we confirmed 95 quasars (including at least two broad absorption line [BAL] quasars), 184 emission-line galaxies, and 12 stars. Of these, 14 quasars were previously confirmed by the $2 \mathrm{dF}$ ( Two Degree Field) survey and by more recent (post-DR1) SDSS spectroscopic follow-up (noted in Table 3); one additional quasar was confirmed by $2 \mathrm{dF}$ alone. This study therefore contributes 80 additional faint quasars in the COSMOS field. A total of 45 targets remain without a secure classification at the conclusion of this study, but only seven objects were completely unidentifiable. Table 3 gives a list of confirmed objects along with redshift measurements where applicable. All object spectra and classifications for confirmed targets are given in Figures 13.1-13.56 in the Appendix, presented in order of right ascension. In all, this small field now has 139 confirmed or probable quasars, one of the highest concentrations of spectroscopically confirmed quasars in the sky.

The two panels of Figure 4 give the SDSS $g$-magnitude differential and cumulative histograms of the target sample, divided into four categories: the full sample for the $2 \mathrm{deg}^{2}$ COSMOS field, the sample contained in the area observed, the objects actually targeted, and the raw quasar yields from our run. In Figure 5 we plot our measured success rate per 1 mag bin; we use a linear fit to predict the number of quasars we would have confirmed if we had been able to cover the full SDSS quasar candidate sample. We expect that if we had been able to target all candidates located within the two pointings, we would have recovered 110 total quasars, 15 of which would overlap the SDSS DR4 list. Of the overlaps, 14 are included in the current study, while one additional object was not observed as part of this work. We project that the total quasar count over the full field using the SDSS selection flags would have been roughly 159, 20 of which would overlap the SDSS DR4 list. Thus, in two pointings and a little over two hours of MMT Hectospec observation time we were able to confirm nearly $60 \%$ of the quasars that had not been previously targeted for spectroscopic follow-up (as of SDSS DR1) down to $g=22.5$ over $2 \mathrm{deg}^{2}$.

Combining our prediction of 110 quasars for the observed pointings with the 25 nonoverlapping quasars confirmed by SDSS, we calculate a surface density of $97 \pm 8$ quasars $\operatorname{deg}^{-2}$ down to SDSS $g=22.5$. Over the entire COSMOS field, we combine our prediction of 159 quasars plus the 44 nonoverlapping quasars confirmed by SDSS and calculate an expected surface density of $102 \pm 7 \mathrm{deg}^{-2}$. These surface densities and the projected quasar yield are lower limits, as some fraction of the unclassified objects may turn out to be quasars. If all 14 QUASAR? objects are in fact quasars, we predict 127 quasars over our two pointings (with 15 SDSS overlaps) and a surface density in the observed portion of the field of $109 \pm 9 \mathrm{deg}^{-2}$. The projected quasar yield would be roughly 184 (with 20 SDSS overlaps) for the entire COSMOS field, resulting in a projected surface density estimate of $114 \pm 8 \mathrm{deg}^{-2}$. Most likely only a fraction of the QUASAR? objects are in fact quasars, so these predictions are upper limits.

Table 4 summarizes these results, while Figure 6 shows the combined differential and cumulative histograms, where we have included our newly confirmed faint SDSS sample and previously confirmed SDSS quasars. For comparison, Richards et al. (2005) present the 2dF-SDSS LRG (luminous red galaxy) and QSO Survey (2SLAQ) cumulative number counts to be $93.8 \mathrm{deg}^{-2}$ down to $g=22$ (magnitude corrected for Galactic extinction) and for a redshift range of $0.3<z<2.2$, limits very similar to our survey. For the same constraints, work by Boyle et al. (2000) corresponds to $79 \mathrm{deg}^{-2}$ and work by Croom et al. (2004) yields $63 \mathrm{deg}^{-2}$. A rough extrapolation of the cumulative quasar histogram of Richards et al. (2005) predicts that we should see between 90 and $100 \mathrm{deg}^{-2}$ down to $g \sim 22.5$, consistent with our results.

Some fraction of the narrow-line objects are likely to be lowluminosity type 2 active galactic nuclei (AGNs). For confirmed emission-line galaxies in our sample at redshifts below 0.38, when the $\mathrm{H} \alpha$ and $\mathrm{N}$ II lines remain within our observed wavelength range, we measure the flux in the [N II] $26584, \mathrm{H} \alpha$, [O III] $\lambda 5007$, and $\mathrm{H} \beta$ emission lines. In Figure 7 we plot the flux ratios on a BPT (Baldwin-Phillips-Televich) diagram (Baldwin et al. 1981). Using the revised relation from Kauffmann et al. (2003) for distinguishing star-forming galaxies and AGNs and a signalto-noise ratio $(\mathrm{S} / \mathrm{N})$ cut of 3 , we find that roughly 30 out of 111 are in fact type 2 objects. We define our $\mathrm{S} / \mathrm{N}$ as the flux in the line divided by the rms scatter in the continuum near the line. This 
TABLE 3

Spectroscopically Confirmed Targets in the COSMOS Field

\begin{tabular}{|c|c|c|c|c|}
\hline Name & $\begin{array}{c}\text { R.A. } \\
(\mathrm{J} 2000.0)\end{array}$ & $\begin{array}{c}\text { Decl. } \\
(\mathrm{J} 2000.0)\end{array}$ & Classification & Redshift $^{\mathrm{a}}$ \\
\hline SDSS J095739.25+020557.9 ......................... & 95739.25 & 20557.98 & GALAXY & $0.322194 \pm 0.000014$ \\
\hline 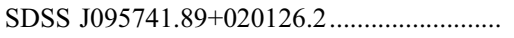 & 95741.89 & 20126.25 & GALAXY & $0.130360 \pm 0.000014$ \\
\hline SDSS J095743.44+015649.3 ….................... & 95743.44 & 15649.38 & GALAXY & $0.575043 \pm 0.000026$ \\
\hline 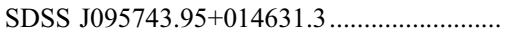 & 95743.95 & 14631.36 & GALAXY & $0.780036 \pm 0.000040$ \\
\hline 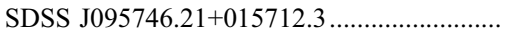 & 95746.21 & 15712.38 & QUASAR & $0.904538 \pm 0.000425$ \\
\hline 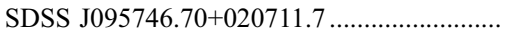 & 95746.70 & 20711.71 & QUASAR & $0.987245 \pm 0.000328$ \\
\hline SDSS J095754.77+015234.6 ........................ & 95754.77 & 15234.68 & GALAXY & $0.194409 \pm 0.000013$ \\
\hline 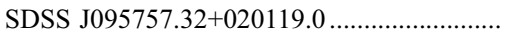 & 95757.32 & 20119.05 & GALAXY & $0.474040 \pm 0.000017$ \\
\hline SDSS J095758.11+020203.5 „...................... & 95758.11 & 20203.51 & GALAXY & $0.209301 \pm 0.000009$ \\
\hline 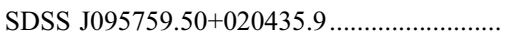 & 95759.50 & 20435.90 & QUASAR & $2.031420 \pm 0.000191^{\mathrm{b}}$ \\
\hline SDSS J095804.25+015526.5 ........................ & 95804.25 & 15526.58 & GALAXY & $0.356245 \pm 0.000012$ \\
\hline SDSS J095804.43+020600.4 ... & 95804.43 & 20600.43 & QUASAR & $1.843120 \pm 0.001279$ \\
\hline SDSS J095805.48+021657.9 ... & 95805.48 & 21657.93 & GALAXY & $0.425338 \pm 0.000006$ \\
\hline SDSS J095806.99+014202.7 ...... & 95806.99 & 14202.77 & QUASAR & $1.710940 \pm 0.000600$ \\
\hline 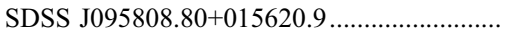 & 95808.80 & 15620.94 & GALAXY & $0.910153 \pm 0.000030$ \\
\hline SDSS J095809.92+021057.6 ........................ & 95809.92 & 21057.61 & QUASAR & $0.836802 \pm 0.000420$ \\
\hline SDSS J095810.80+014218.7 ...... & 95810.80 & 14218.72 & STAR & $\ldots$ \\
\hline SDSS J095811.08+013845.3 ....... & 95811.08 & 13845.38 & GALAXY & $0.393641 \pm 0.000030$ \\
\hline SDSS J095812.83+015525.0 ........ & 95812.83 & 15525.03 & GALAXY & $0.382592 \pm 0.000020$ \\
\hline SDSS J095813.31+013509.8 ....... & 95813.31 & 13509.88 & GALAXY & $0.124726 \pm 0.000019$ \\
\hline SDSS J095813.34+020536.0 ...... & 95813.34 & 20536.02 & QUASAR & $0.702752 \pm 0.000482$ \\
\hline SDSS J095815.29+014738.4 „....................... & 95815.29 & 14738.47 & QUASAR & $2.212010 \pm 0.001236$ \\
\hline 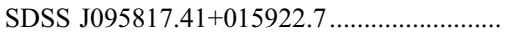 & 95817.41 & 15922.77 & GALAXY & $0.309371 \pm 0.000018$ \\
\hline 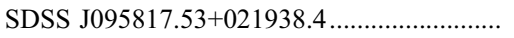 & 95817.53 & 21938.46 & QUASAR & $0.730052 \pm 0.000242$ \\
\hline SDSS J095820.78+020213.4 ......................... & 95820.78 & 20213.41 & QUASAR & $1.856180 \pm 0.000441$ \\
\hline 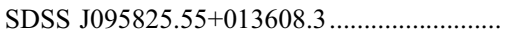 & 95825.55 & 13608.31 & GALAXY & $0.322467 \pm 0.000022$ \\
\hline 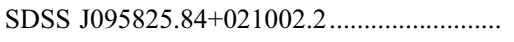 & 95825.84 & 21002.24 & GALAXY & $0.676363 \pm 0.000042$ \\
\hline SDSS J095827.76+014136.2 ....... & 95827.76 & 14136.27 & GALAXY & $0.006000 \pm 0.000006$ \\
\hline 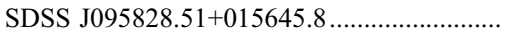 & 95828.51 & 15645.85 & GALAXY & $0.375036 \pm 0.000027$ \\
\hline 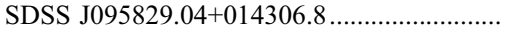 & 95829.04 & 14306.85 & GALAXY & $0.733573 \pm 0.000066$ \\
\hline 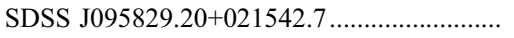 & 95829.20 & 21542.73 & QUASAR & $0.945722 \pm 0.000609$ \\
\hline 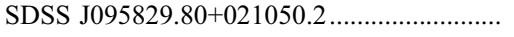 & 95829.80 & 21050.26 & QUASAR & $1.188000 \pm 0.000316$ \\
\hline 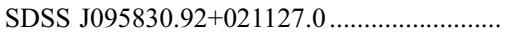 & 95830.92 & 21127.06 & GALAXY & $0.700667 \pm 0.000096$ \\
\hline SDSS J095830.96+013956.5 „....................... & 95830.96 & 13956.59 & GALAXY & $0.671713 \pm 0.000039$ \\
\hline SDSS J095831.05+020153.3 …..................... & 95831.05 & 20153.32 & GALAXY & $0.283694 \pm 0.000011$ \\
\hline SDSS J095837.65+015103.1 ........................ & 95837.65 & 15103.16 & GALAXY & $0.310563 \pm 0.000028$ \\
\hline SDSS J095837.73+015144.5 „...................... & 95837.73 & 15144.56 & GALAXY & $0.397999 \pm 0.000017$ \\
\hline 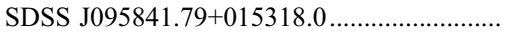 & 95841.79 & 15318.06 & QUASAR & $1.783840 \pm 0.001197$ \\
\hline SDSS J095842.03+015857.7 ......................... & 95842.03 & 15857.72 & QUASAR & $1.333690 \pm 0.000931$ \\
\hline 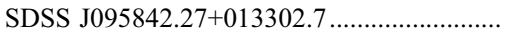 & 95842.27 & 13302.77 & GALAXY & $0.633906 \pm 0.000070$ \\
\hline SDSS J095845.32+022309.5 „....................... & 95845.32 & 22309.56 & STAR & $\ldots$ \\
\hline 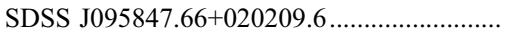 & 95847.66 & 20209.60 & GALAXY & $0.340087 \pm 0.000031$ \\
\hline SDSS J095848.54+014922.4 ........................ & 95848.54 & 14922.40 & GALAXY & $0.367708 \pm 0.000027$ \\
\hline SDSS J095851.50+015944.2 „........................ & 95851.50 & 15944.26 & GALAXY & $0.202467 \pm 0.000030$ \\
\hline SDSS J095851.63+020020.6 ....................... & 95851.63 & 20020.66 & GALAXY & $0.517379 \pm 0.000070$ \\
\hline SDSS J095852.45+021205.5 „...................... & 95852.45 & 21205.54 & GALAXY & $0.379686 \pm 0.000034$ \\
\hline SDSS J095854.01+015601.6 ....................... & 95854.01 & 15601.68 & GALAXY & $0.132268 \pm 0.000010$ \\
\hline 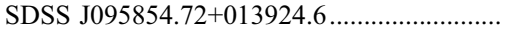 & 95854.72 & 13924.66 & GALAXY & $0.130793 \pm 0.000032$ \\
\hline SDSS J095855.23+013509.3 …................... & 95855.23 & 13509.38 & GALAXY & $0.623221 \pm 0.000029$ \\
\hline SDSS J095856.27+014312.8 ......................... & 95856.27 & 14312.82 & GALAXY & $0.350809 \pm 0.000019$ \\
\hline SDSS J095856.52+021255.2 ........................ & 95856.52 & 21255.22 & GALAXY & $0.601531 \pm 0.000022$ \\
\hline SDSS J095857.35+020137.6 ........................ & 95857.35 & 20137.63 & GALAXY & $0.241260 \pm 0.000011$ \\
\hline SDSS J095858.38+014705.3 …................... & 95858.38 & 14705.38 & GALAXY & $0.443308 \pm 0.000025$ \\
\hline SDSS J095858.43+015727.8 ….................... & 95858.43 & 15727.86 & GALAXY & $0.187933 \pm 0.000042$ \\
\hline SDSS J095902.55+022511.4 „....................... & 95902.55 & 22511.42 & QUASAR & $1.106050 \pm 0.000783$ \\
\hline 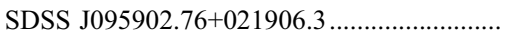 & 95902.76 & 21906.34 & QUASAR & $0.345449 \pm 0.000013^{\mathrm{b}}$ \\
\hline SDSS J095903.23+022002.9 …................... & 95903.23 & 22002.90 & QUASAR & $1.138820 \pm 0.000595$ \\
\hline 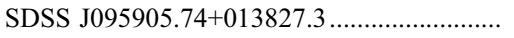 & 95905.74 & 13827.31 & GALAXY & $0.424361 \pm 0.000014$ \\
\hline SDSS J095906.46+013219.1 ......................... & 95906.46 & 13219.14 & GALAXY & $0.364954 \pm 0.000015$ \\
\hline SDSS J095907.65+020820.6 ......................... & 95907.65 & 20820.65 & QUASAR & $0.354505 \pm 0.000033$ \\
\hline SDSS J095912.18+020052.5 „...................... & 95912.18 & 20052.59 & GALAXY & $0.399436 \pm 0.000016$ \\
\hline SDSS J095913.74+022222.8 ….................... & 95913.74 & 22222.80 & GALAXY & $0.186780 \pm 0.000020$ \\
\hline 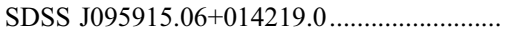 & 95915.06 & 14219.04 & GALAXY & $0.445974 \pm 0.000013$ \\
\hline SDSS J095915.88+014430.2 „...................... & 95915.88 & 14430.26 & GALAXY & $0.531081 \pm 0.000012$ \\
\hline
\end{tabular}


TABLE 3-Continued

\begin{tabular}{|c|c|c|c|c|}
\hline Name & $\begin{array}{c}\text { R.A. } \\
\text { (J2000.0) }\end{array}$ & $\begin{array}{c}\text { Decl. } \\
(\mathrm{J} 2000.0)\end{array}$ & Classification & Redshift $^{\mathrm{a}}$ \\
\hline 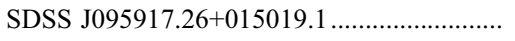 & 95917.26 & 15019.14 & QUASAR & $1.342660 \pm 0.000246$ \\
\hline 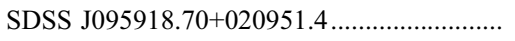 & 95918.70 & 20951.48 & QUASAR & $1.161600 \pm 0.000345^{\mathrm{b}}$ \\
\hline 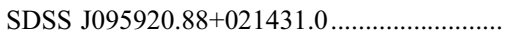 & 95920.88 & 21431.05 & GALAXY & $0.303490 \pm 0.000058$ \\
\hline 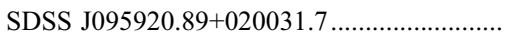 & 95920.89 & 20031.71 & QUASAR & $1.483000 \pm 0.000999$ \\
\hline 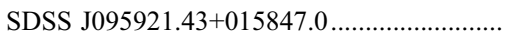 & 95921.43 & 15847.06 & GALAXY & $0.109154 \pm 0.000014$ \\
\hline 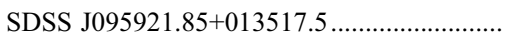 & 95921.85 & 13517.59 & GALAXY & $0.186926 \pm 0.000028$ \\
\hline 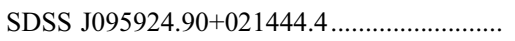 & 95924.90 & 21444.41 & GALAXY & $0.344956 \pm 0.000030$ \\
\hline 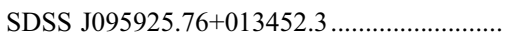 & 95925.76 & 13452.35 & STAR & \\
\hline 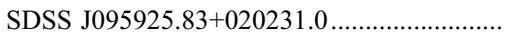 & 95925.83 & 20231.09 & GALAXY & $0.252403 \pm 0.000013$ \\
\hline 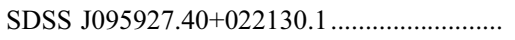 & 95927.40 & 22130.13 & GALAXY & $0.133125 \pm 0.000018$ \\
\hline 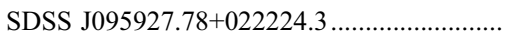 & 95927.78 & 22224.34 & GALAXY & $0.359376 \pm 0.000017$ \\
\hline 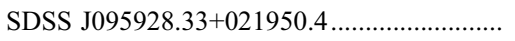 & 95928.33 & 21950.44 & QUASAR & $1.478290 \pm 0.000538$ \\
\hline 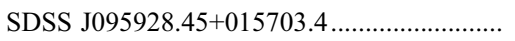 & 95928.45 & 15703.42 & GALAXY & $0.328650 \pm 0.000012$ \\
\hline 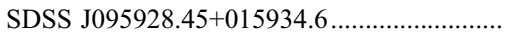 & 95928.45 & 15934.62 & QUASAR & $1.165960 \pm 0.000671$ \\
\hline 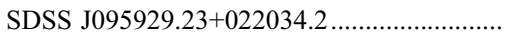 & 95929.23 & 22034.22 & QUASAR & $1.738060 \pm 0.001059$ \\
\hline 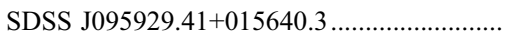 & 95929.41 & 15640.34 & GALAXY & $0.561256 \pm 0.000023$ \\
\hline 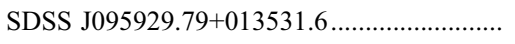 & 95929.79 & 13531.63 & GALAXY & $0.758662 \pm 0.000018$ \\
\hline 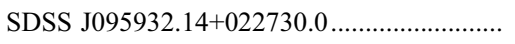 & 95932.14 & 22730.02 & GALAXY & $0.332977 \pm 0.000018$ \\
\hline 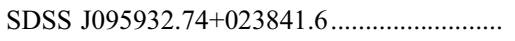 & 95932.74 & 23841.64 & GALAXY & $0.246618 \pm 0.000027$ \\
\hline 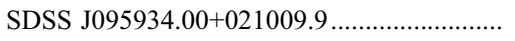 & 95934.00 & 21009.94 & GALAXY & $0.186060 \pm 0.000008$ \\
\hline 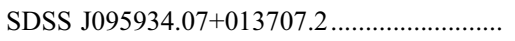 & 95934.07 & 13707.28 & GALAXY & $0.032031 \pm 0.000010$ \\
\hline 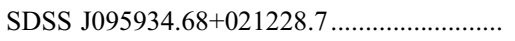 & 95934.68 & 21228.76 & GALAXY & $0.345155 \pm 0.000017$ \\
\hline 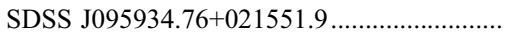 & 95934.76 & 21551.94 & GALAXY & $0.594848 \pm 0.000031$ \\
\hline SDSS J095934.89+021422.0 & 95934.89 & 21422.09 & QUASAR & $1.733600 \pm 0.000768$ \\
\hline 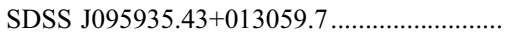 & 95935.43 & 13059.79 & QUASAR & $1.668750 \pm 0.000723$ \\
\hline 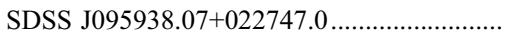 & 95938.07 & 22747.01 & GALAXY & $0.426893 \pm 0.000025$ \\
\hline 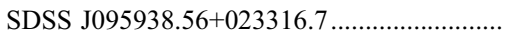 & 95938.56 & 23316.77 & QUASAR & $0.753602 \pm 0.000090$ \\
\hline 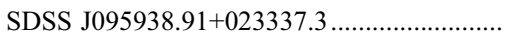 & 95938.91 & 23337.36 & GALAXY & $0.412420 \pm 0.000072$ \\
\hline 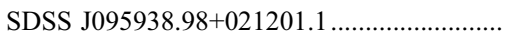 & 95938.98 & 21201.18 & QUASAR & $0.688950 \pm 0.000255$ \\
\hline 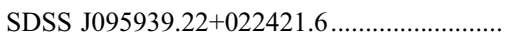 & 95939.22 & 22421.67 & GALAXY & $0.224803 \pm 0.000035$ \\
\hline 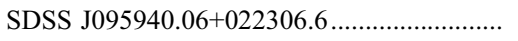 & 95940.06 & 22306.64 & QUASAR & $1.123300 \pm 0.000160$ \\
\hline 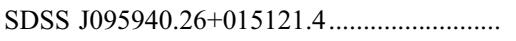 & 95940.26 & 15121.49 & GALAXY & $0.250914 \pm 0.000006$ \\
\hline 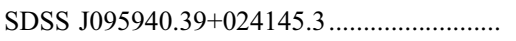 & 95940.39 & 24145.38 & GALAXY & $0.358390 \pm 0.000007$ \\
\hline 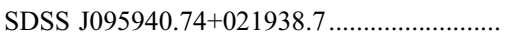 & 95940.74 & 21938.71 & QUASAR & $1.459100 \pm 0.000378$ \\
\hline 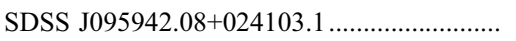 & 95942.08 & 24103.12 & QUASAR & $1.795 \pm 0.002^{\mathrm{c}}$ \\
\hline 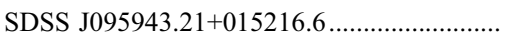 & 95943.21 & 15216.60 & GALAXY & $0.229967 \pm 0.000034$ \\
\hline 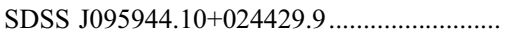 & 95944.10 & 24429.97 & GALAXY & $0.464817 \pm 0.000013$ \\
\hline 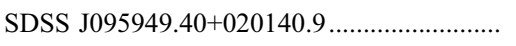 & 95949.40 & 20140.98 & QUASAR & $1.753520 \pm 0.000451^{\mathrm{b}}$ \\
\hline SDSS J095953.55+022738.9 …….................. & 95953.55 & 22738.98 & GALAXY & $0.082599 \pm 0.000004$ \\
\hline SDSS J095954.78+013206.4 ........................... & 95954.78 & 13206.43 & QUASAR & $0.481540 \pm 0.000062$ \\
\hline 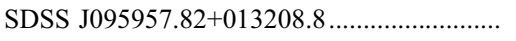 & 95957.82 & 13208.88 & GALAXY & $0.434306 \pm 0.000035$ \\
\hline 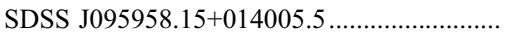 & 95958.15 & 14005.59 & GALAXY & $0.091670 \pm 0.000004$ \\
\hline 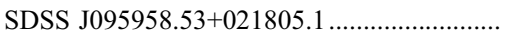 & 95958.53 & 21805.18 & QUASAR & $1.788850 \pm 0.000958$ \\
\hline 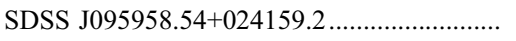 & 95958.54 & 24159.24 & GALAXY & $0.728646 \pm 0.000024$ \\
\hline SDSS J095959.11+014941.5 .......................... & 95959.11 & 14941.59 & GALAXY & $0.359542 \pm 0.000043$ \\
\hline SDSS J100000.17+015606.8 ........................... & 100000.17 & 15606.86 & GALAXY & $0.371913 \pm 0.000023$ \\
\hline 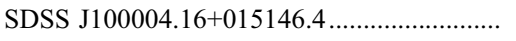 & 100004.16 & 15146.40 & GALAXY & $0.266362 \pm 0.000018$ \\
\hline 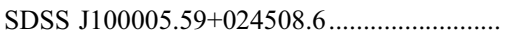 & 100005.59 & 24508.60 & QUASAR & $0.500300 \pm 0.000115$ \\
\hline 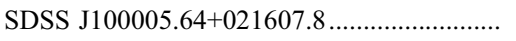 & 100005.64 & 21607.89 & GALAXY & $0.236488 \pm 0.000009$ \\
\hline 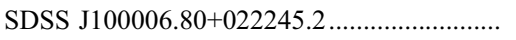 & 100006.80 & 22245.26 & GALAXY & $0.011757 \pm 0.000005$ \\
\hline 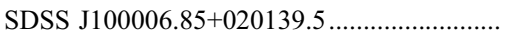 & 100006.85 & 20139.54 & GALAXY & $0.309486 \pm 0.000034$ \\
\hline 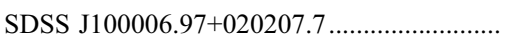 & 100006.97 & 20207.72 & GALAXY & $0.314517 \pm 0.000012$ \\
\hline 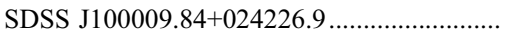 & 100009.84 & 24226.92 & GALAXY & $0.600963 \pm 0.000009$ \\
\hline 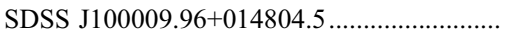 & 100009.96 & 14804.50 & GALAXY & $0.267040 \pm 0.000025$ \\
\hline 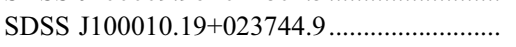 & 100010.19 & 23744.94 & QUASAR & $1.560 \pm 0.002^{\mathrm{d}}$ \\
\hline 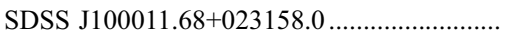 & 100011.68 & 23158.08 & GALAXY & $0.332432 \pm 0.000018$ \\
\hline 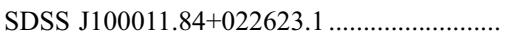 & 100011.84 & 22623.17 & GALAXY & $0.440452 \pm 0.000007$ \\
\hline SDSS J100012.08+014439.8 ……..................... & 100012.08 & 14439.87 & QUASAR & $1.148090 \pm 0.000800$ \\
\hline 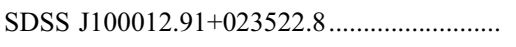 & 100012.91 & 23522.81 & QUASAR & $0.701555 \pm 0.000076^{\mathrm{b}}$ \\
\hline 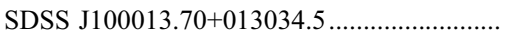 & 100013.70 & 13034.59 & QUASAR & $0.852805 \pm 0.000240$ \\
\hline SDSS J100014.09+022838.5 ............................ & 100014.09 & 22838.56 & QUASAR & $1.253000 \pm 0.000634$ \\
\hline 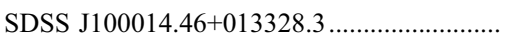 & 100014.46 & 13328.33 & GALAXY & $0.344993 \pm 0.000017$ \\
\hline 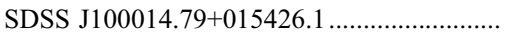 & 100014.79 & 15426.10 & GALAXY & $0.670886 \pm 0.000043$ \\
\hline SDSS J100016.25+022450.8 …........................ & 100016.25 & 22450.83 & GALAXY & $0.350322 \pm 0.000014$ \\
\hline 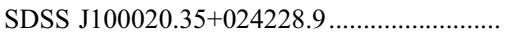 & 100020.35 & 24228.90 & GALAXY & $0.250147 \pm 0.000026$ \\
\hline
\end{tabular}


TABLE 3-Continued

\begin{tabular}{|c|c|c|c|c|}
\hline Name & $\begin{array}{c}\text { R.A. } \\
\text { (J2000.0) }\end{array}$ & $\begin{array}{c}\text { Decl. } \\
(\mathrm{J} 2000.0)\end{array}$ & Classification & Redshift $^{\mathrm{a}}$ \\
\hline SDSS J100021.74+015009.4 & 100021.74 & 15009.42 & GALAXY & $0.218412 \pm 0.000022$ \\
\hline SDSS J100022.45+023018.0 ……………........ & 100022.45 & 23018.03 & GALAXY & $0.268133 \pm 0.000022$ \\
\hline SDSS J100023.31+023712.3 ………................ & 100023.31 & 23712.39 & GALAXY & $0.291524 \pm 0.000022$ \\
\hline 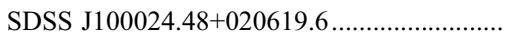 & 100024.48 & 20619.62 & QUASAR & $2.288520 \pm 0.000576$ \\
\hline SDSS J100024.64+023149.0 & 100024.64 & 23149.04 & QUASAR & $1.311200 \pm 0.000179^{\mathrm{b}}$ \\
\hline SDSS J100024.71+025039.0 & 100024.71 & 25039.01 & GALAXY & $0.696753 \pm 0.000051$ \\
\hline SDSS J100025.06+024128.4 & 100025.06 & 24128.46 & QUASAR & $1.881820 \pm 0.000839$ \\
\hline SDSS J100025.25+015852.0 & 100025.25 & 15852.06 & QUASAR & $0.372559 \pm 0.000021^{\mathrm{b}}$ \\
\hline 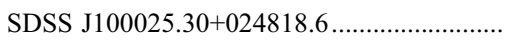 & 100025.30 & 24818.68 & GALAXY & $0.331828 \pm 0.000016$ \\
\hline 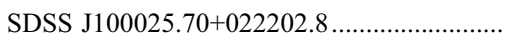 & 100025.70 & 22202.85 & GALAXY & $0.340500 \pm 0.000012$ \\
\hline SDSS J100026.56+014538.9 …………..... & 100026.56 & 14538.98 & GALAXY & $0.569300 \pm 0.000234$ \\
\hline SDSS J100027.75+015703.9 …………..... & 100027.75 & 15703.99 & GALAXY & $0.264465 \pm 0.000007$ \\
\hline SDSS J100029.07+022850.0 & 100029.07 & 22850.05 & GALAXY & $0.688393 \pm 0.000050$ \\
\hline SDSS J100030.47+023735.5 & 100030.47 & 23735.54 & QUASAR & $1.839460 \pm 0.000614$ \\
\hline 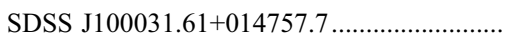 & 100031.61 & 14757.73 & QUASAR & $1.679100 \pm 0.001215$ \\
\hline SDSS J100033.19+015038.4 …........................ & 100033.19 & 15038.47 & GALAXY & $0.571064 \pm 0.000078$ \\
\hline SDSS J100033.39+015237.0 & 100033.39 & 15237.05 & QUASAR & $0.832222 \pm 0.000273$ \\
\hline 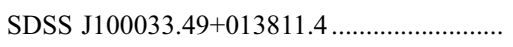 & 100033.49 & 13811.40 & QUASAR & $0.520990 \pm 0.000207$ \\
\hline 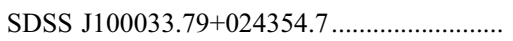 & 100033.79 & 24354.76 & QUASAR & $1.310500 \pm 0.000667$ \\
\hline 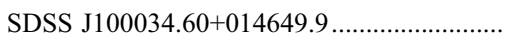 & 100034.60 & 14649.98 & GALAXY & $0.184455 \pm 0.000031$ \\
\hline SDSS J100034.93+020235.0 & 100034.93 & 20235.01 & QUASAR & $1.179840 \pm 0.000828$ \\
\hline 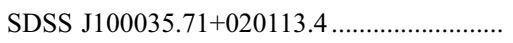 & 100035.71 & 20113.44 & GALAXY & $0.265439 \pm 0.000010$ \\
\hline SDSS J100035.86+022653.5 ........................... & 100035.86 & 22653.55 & GALAXY & $0.338454 \pm 0.000009$ \\
\hline 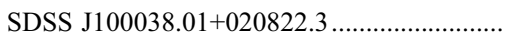 & 100038.01 & 20822.38 & QUASAR & $1.825 \pm 0.002^{\mathrm{c}}$ \\
\hline SDSS J100040.15+024751.5 & 100040.15 & 24751.50 & QUASAR & $1.041570 \pm 0.000271$ \\
\hline SDSS J100040.70+024934.9 ……………........ & 100040.70 & 24934.93 & GALAXY & $0.416288 \pm 0.000031$ \\
\hline SDSS J100041.42+021331.8 ……………....... & 100041.42 & 21331.87 & GALAXY & $0.325041 \pm 0.000011$ \\
\hline SDSS J100042.09+022533.9.......................... & 100042.09 & 22533.96 & GALAXY & $0.311771 \pm 0.000009$ \\
\hline 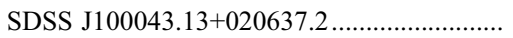 & 100043.13 & 20637.22 & QUASAR & $0.360599 \pm 0.000039^{\mathrm{b}}$ \\
\hline 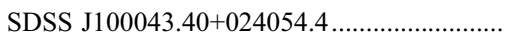 & 100043.40 & 24054.44 & GALAXY & $0.305230 \pm 0.000019$ \\
\hline 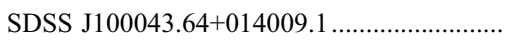 & 100043.64 & 14009.19 & QUASAR & $2.029420 \pm 0.000349$ \\
\hline 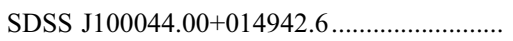 & 100044.00 & 14942.63 & GALAXY & $0.527891 \pm 0.000020$ \\
\hline 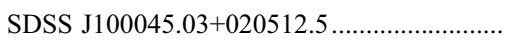 & 100045.03 & 20512.55 & STAR & $\ldots$ \\
\hline 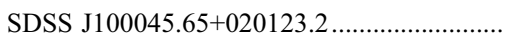 & 100045.65 & 20123.26 & GALAXY & $0.092532 \pm 0.000004$ \\
\hline SDSS J100046.73+020404.4 & 100046.73 & 20404.44 & QUASAR & $0.553653 \pm 0.000166$ \\
\hline 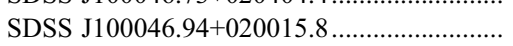 & 100046.94 & 20015.87 & QUASAR & $1.920230 \pm 0.000707^{\mathrm{b}}$ \\
\hline 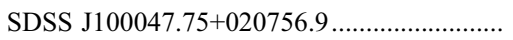 & 100047.75 & 20756.96 & QUASAR & $2.160590 \pm 0.001104$ \\
\hline 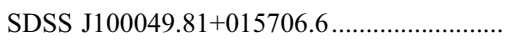 & 100049.81 & 15706.62 & GALAXY & $0.361678 \pm 0.000029$ \\
\hline SDSS J100050.72+024925.1 ……................... & 100050.72 & 24925.10 & STAR & $\ldots$ \\
\hline SDSS J100050.95+024753.5 …………........... & 100050.95 & 24753.59 & GALAXY & $0.429036 \pm 0.000018$ \\
\hline 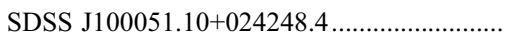 & 100051.10 & 24248.49 & GALAXY & $0.186305 \pm 0.000023$ \\
\hline 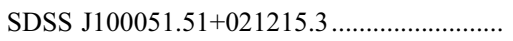 & 100051.51 & 21215.30 & QUASAR & $1.850060 \pm 0.000298$ \\
\hline SDSS J100051.93+015919.2 ………….......... & 100051.93 & 15919.28 & QUASAR & $2.239610 \pm 0.001088$ \\
\hline 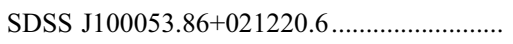 & 100053.86 & 21220.62 & GALAXY & $0.121853 \pm 0.000008$ \\
\hline SDSS J100054.84+022623.4 & 100054.84 & 22623.46 & GALAXY & $0.464736 \pm 0.000017$ \\
\hline 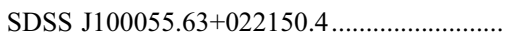 & 100055.63 & 22150.40 & QUASAR & $1.933090 \pm 0.000419$ \\
\hline 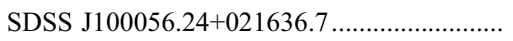 & 100056.24 & 21636.73 & GALAXY & $0.123186 \pm 0.000013$ \\
\hline SDSS J100056.59+025129.5 ……………....... & 100056.59 & 25129.55 & GALAXY & $0.510724 \pm 0.000021$ \\
\hline SDSS J100058.33+015208.5 ………….......... & 100058.33 & 15208.58 & QUASAR & $2.024160 \pm 0.001954$ \\
\hline SDSS J100058.46+015436.9.......................... & 100058.46 & 15436.97 & GALAXY & $0.373979 \pm 0.000032$ \\
\hline SDSS J100058.70+022556.2 …………........... & 100058.70 & 22556.20 & QUASAR & $0.695149 \pm 0.000384$ \\
\hline 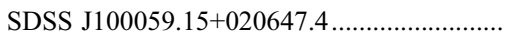 & 100059.15 & 20647.48 & STAR & $\ldots$ \\
\hline SDSS J100059.49+021535.4 & 100059.49 & 21535.49 & STAR & \\
\hline 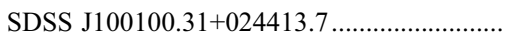 & 100100.31 & 24413.74 & QUASAR & $2.172130 \pm 0.000510$ \\
\hline 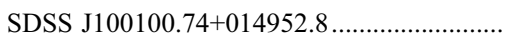 & 100100.74 & 14952.89 & GALAXY & $0.528999 \pm 0.000048$ \\
\hline 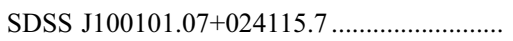 & 100101.07 & 24115.79 & GALAXY & $0.346603 \pm 0.000024$ \\
\hline SDSS J100104.07+023442.6........................... & 100104.07 & 23442.63 & STAR & $\ldots$ \\
\hline 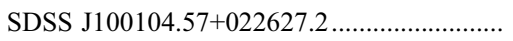 & 100104.57 & 22627.20 & GALAXY & $0.347905 \pm 0.000038$ \\
\hline SDSS J100104.67+025251.8 & 100104.67 & 25251.85 & GALAXY & $0.452569 \pm 0.000028$ \\
\hline 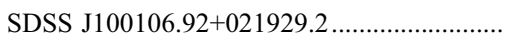 & 100106.92 & 21929.20 & GALAXY & $0.338016 \pm 0.000017$ \\
\hline 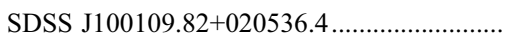 & 100109.82 & 20536.49 & GALAXY & $0.282967 \pm 0.000013$ \\
\hline SDSS J100110.72+022049.1 ………............. & 100110.72 & 22049.12 & GALAXY & $0.248073 \pm 0.000005$ \\
\hline 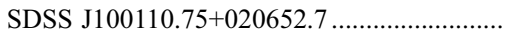 & 100110.75 & 20652.70 & GALAXY & $0.247533 \pm 0.000032$ \\
\hline 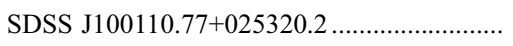 & 100110.77 & 25320.25 & GALAXY & $0.103 \pm 0.002^{\mathrm{d}}$ \\
\hline 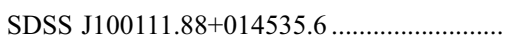 & 100111.88 & 14535.64 & GALAXY & $0.339773 \pm 0.000051$ \\
\hline
\end{tabular}


TABLE 3-Continued

\begin{tabular}{|c|c|c|c|c|}
\hline Name & $\begin{array}{c}\text { R.A. } \\
\text { (J2000.0) }\end{array}$ & $\begin{array}{c}\text { Decl. } \\
(\mathrm{J} 2000.0)\end{array}$ & Classification & Redshift $^{\mathrm{a}}$ \\
\hline 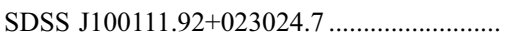 & 100111.92 & 23024.76 & QUASAR & $1.494800 \pm 0.000513$ \\
\hline SDSS J100112.24+015842.2 ……..................... & 100112.24 & 15842.24 & GALAXY & $0.388559 \pm 0.000025$ \\
\hline 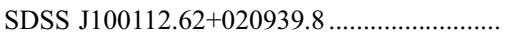 & 100112.62 & 20939.85 & QUASAR & $1.817170 \pm 0.000492$ \\
\hline 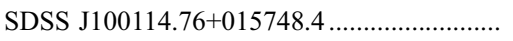 & 100114.76 & 15748.49 & GALAXY & $0.360802 \pm 0.000033$ \\
\hline 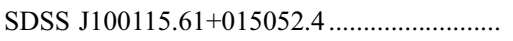 & 100115.61 & 15052.44 & GALAXY & $0.567047 \pm 0.000060$ \\
\hline 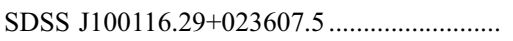 & 100116.29 & 23607.52 & QUASAR & $0.959422 \pm 0.000644$ \\
\hline 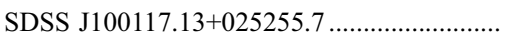 & 100117.13 & 25255.77 & GALAXY & $0.694750 \pm 0.000073$ \\
\hline 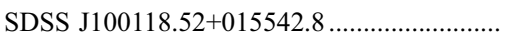 & 100118.52 & 15542.81 & QUASAR & $0.528661 \pm 0.000045$ \\
\hline SDSS J100118.57+022958.0 & 100118.57 & 22958.05 & GALAXY & $0.217207 \pm 0.000013$ \\
\hline SDSS J100118.62+021431.1 … & 100118.62 & 21431.12 & STAR & $\ldots$ \\
\hline SDSS J100119.32+024559.6 & 100119.32 & 24559.68 & GALAXY & $0.073011 \pm 0.000007$ \\
\hline SDSS J100120.18+023047.7 _... & 100120.18 & 23047.70 & GALAXY & $0.176016 \pm 0.000009$ \\
\hline 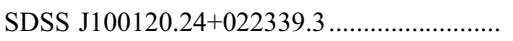 & 100120.24 & 22339.37 & GALAXY & $0.247721 \pm 0.000014$ \\
\hline 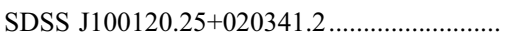 & 100120.25 & 20341.25 & QUASAR & $0.905615 \pm 0.000289$ \\
\hline 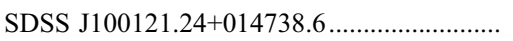 & 100121.24 & 14738.61 & GALAXY & $0.339145 \pm 0.000026$ \\
\hline 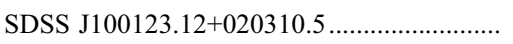 & 100123.12 & 20310.58 & GALAXY & $0.460211 \pm 0.000025$ \\
\hline 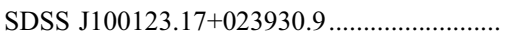 & 100123.17 & 23930.96 & GALAXY & $0.219494 \pm 0.000023$ \\
\hline 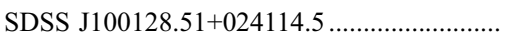 & 100128.51 & 24114.53 & GALAXY & $0.419649 \pm 0.000087$ \\
\hline 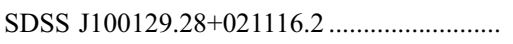 & 100129.28 & 21116.29 & GALAXY & $0.569147 \pm 0.000085$ \\
\hline 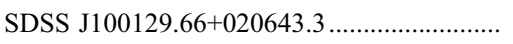 & 100129.66 & 20643.30 & QUASAR & $1.916270 \pm 0.001279$ \\
\hline 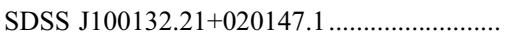 & 100132.21 & 20147.10 & GALAXY & $0.501262 \pm 0.000026$ \\
\hline 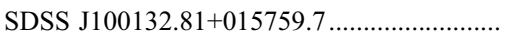 & 100132.81 & 15759.79 & QUASAR & $1.529660 \pm 0.000598$ \\
\hline 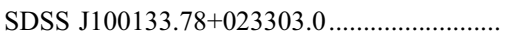 & 100133.78 & 23303.06 & GALAXY & $0.112673 \pm 0.000014$ \\
\hline 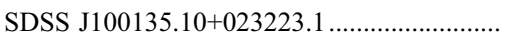 & 100135.10 & 23223.10 & GALAXY & $0.113566 \pm 0.000034$ \\
\hline 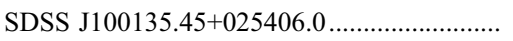 & 100135.45 & 25406.08 & QUASAR & $1.642370 \pm 0.001126$ \\
\hline SDSS J100135.58+024341.5 ............................ & 100135.58 & 24341.52 & GALAXY & $0.916410 \pm 0.000055$ \\
\hline SDSS J100136.50+025303.5 ............................. & 100136.50 & 25303.58 & QUASAR & $2.112940 \pm 0.000625^{\mathrm{b}}$ \\
\hline 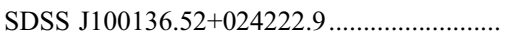 & 100136.52 & 24222.96 & GALAXY & $0.487841 \pm 0.000028$ \\
\hline 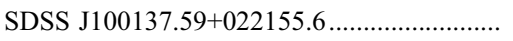 & 100137.59 & 22155.69 & QUASAR & $2.039950 \pm 0.001609$ \\
\hline 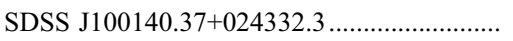 & 100140.37 & 24332.37 & GALAXY & $0.223378 \pm 0.000028$ \\
\hline 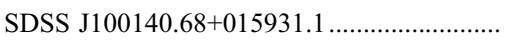 & 100140.68 & 15931.12 & GALAXY & $0.266793 \pm 0.000032$ \\
\hline 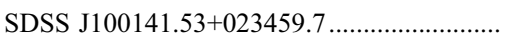 & 100141.53 & 23459.73 & GALAXY & $0.822187 \pm 0.000045$ \\
\hline SDSS J100141.84+020200.2 ........................... & 100141.84 & 20200.20 & GALAXY & $0.684676 \pm 0.000037$ \\
\hline 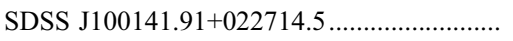 & 100141.91 & 22714.50 & GALAXY & $0.174935 \pm 0.000042$ \\
\hline 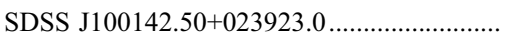 & 100142.50 & 23923.04 & GALAXY & $0.550259 \pm 0.000034$ \\
\hline 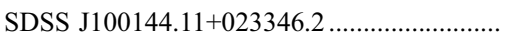 & 100144.11 & 23346.22 & GALAXY & $0.403245 \pm 0.000017$ \\
\hline SDSS J100144.48+021626.9 …...................... & 100144.48 & 21626.97 & GALAXY & $0.474159 \pm 0.000013$ \\
\hline 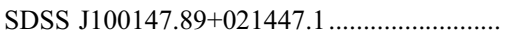 & 100147.89 & 21447.14 & QUASAR & $0.881799 \pm 0.000288$ \\
\hline 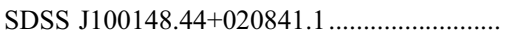 & 100148.44 & 20841.17 & QUASAR & $1.300850 \pm 0.000722$ \\
\hline 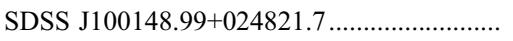 & 100148.99 & 24821.70 & QUASAR & $1.612920 \pm 0.000665$ \\
\hline 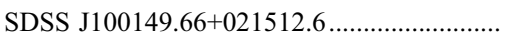 & 100149.66 & 21512.60 & GALAXY & $0.424917 \pm 0.000024$ \\
\hline SDSS J100150.08+024150.6 ............................ & 100150.08 & 24150.60 & GALAXY & $0.195230 \pm 0.000010$ \\
\hline 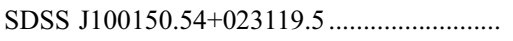 & 100150.54 & 23119.56 & GALAXY & $0.329404 \pm 0.000039$ \\
\hline 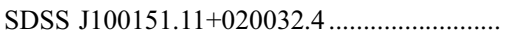 & 100151.11 & 20032.43 & QUASAR & $0.966629 \pm 0.000297$ \\
\hline 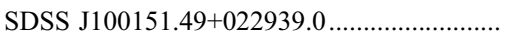 & 100151.49 & 22939.04 & GALAXY & $0.187481 \pm 0.000055$ \\
\hline 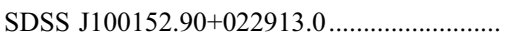 & 100152.90 & 22913.05 & GALAXY & $0.434961 \pm 0.000015$ \\
\hline 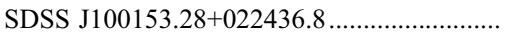 & 100153.28 & 22436.82 & QUASAR & $0.666530 \pm 0.000477$ \\
\hline 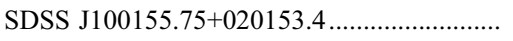 & 100155.75 & 20153.47 & GALAXY & $0.320069 \pm 0.000023$ \\
\hline 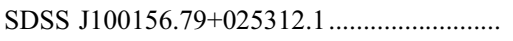 & 100156.79 & 25312.19 & GALAXY & $0.624114 \pm 0.000187$ \\
\hline 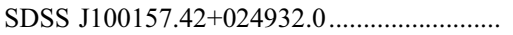 & 100157.42 & 24932.01 & GALAXY & $0.522543 \pm 0.000018$ \\
\hline 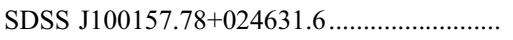 & 100157.78 & 24631.65 & QUASAR & $1.449 \pm 0.002^{\mathrm{d}}$ \\
\hline 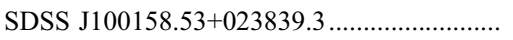 & 100158.53 & 23839.37 & GALAXY & $0.195412 \pm 0.000038$ \\
\hline 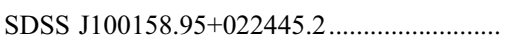 & 100158.95 & 22445.21 & QUASAR & $1.370600 \pm 0.001008$ \\
\hline 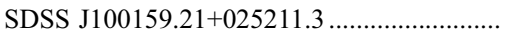 & 100159.21 & 25211.35 & GALAXY & $0.744574 \pm 0.000021$ \\
\hline 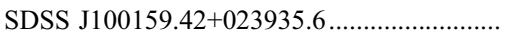 & 100159.42 & 23935.64 & QUASAR & $0.851119 \pm 0.000481$ \\
\hline 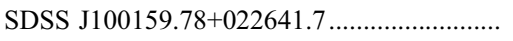 & 100159.78 & 22641.71 & QUASAR & $2.032930 \pm 0.000318$ \\
\hline 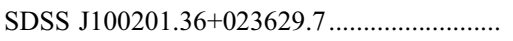 & 100201.36 & 23629.77 & GALAXY & $0.213058 \pm 0.000017$ \\
\hline 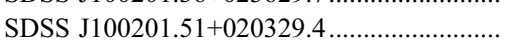 & 100201.51 & 20329.48 & QUASAR & $2.060500 \pm 0.000159^{\mathrm{b}}$ \\
\hline 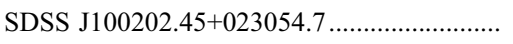 & 100202.45 & 23054.79 & GALAXY & $0.219060 \pm 0.000032$ \\
\hline 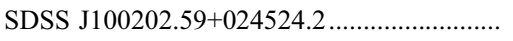 & 100202.59 & 24524.26 & GALAXY & $0.675794 \pm 0.000019$ \\
\hline 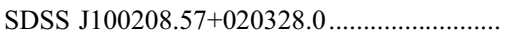 & 100208.57 & 20328.08 & QUASAR & $1.199730 \pm 0.000286$ \\
\hline 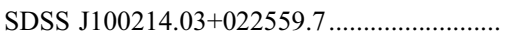 & 100214.03 & 22559.70 & GALAXY & $0.501816 \pm 0.000037$ \\
\hline 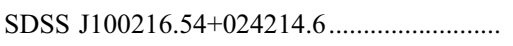 & 100216.54 & 24214.65 & GALAXY & $0.351628 \pm 0.000024$ \\
\hline 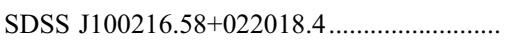 & 100216.58 & 22018.49 & GALAXY & $0.394484 \pm 0.000023$ \\
\hline 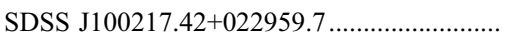 & 100217.42 & 22959.74 & QUASAR & $1.106370 \pm 0.000489$ \\
\hline
\end{tabular}


TABLE 3-Continued

\begin{tabular}{|c|c|c|c|c|}
\hline Name & $\begin{array}{c}\text { R.A. } \\
(\mathrm{J} 2000.0)\end{array}$ & $\begin{array}{c}\text { Decl. } \\
(\mathrm{J} 2000.0)\end{array}$ & Classification & Redshift $^{\mathrm{a}}$ \\
\hline SDSS J100217.45+023545.9 ……................. & 100217.45 & 23545.96 & GALAXY & $0.220081 \pm 0.000027$ \\
\hline 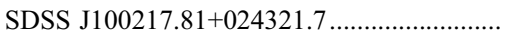 & 100217.81 & 24321.79 & GALAXY & $0.331244 \pm 0.000037$ \\
\hline SDSS J100219.01+022242.4 ………............... & 100219.01 & 22242.42 & GALAXY & $0.222120 \pm 0.000013$ \\
\hline SDSS J100219.42+025049.5 & 100219.42 & 25049.52 & QUASAR & $1.450950 \pm 0.000218$ \\
\hline 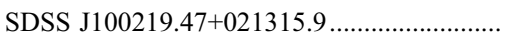 & 100219.47 & 21315.96 & QUASAR & $2.027330 \pm 0.000467$ \\
\hline SDSS J100221.45+022938.7 & 100221.45 & 22938.76 & GALAXY & $0.259461 \pm 0.000011$ \\
\hline 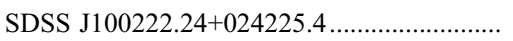 & 100222.24 & 24225.48 & GALAXY & $0.323868 \pm 0.000030$ \\
\hline SDSS J100225.74+024028.0 & 100225.74 & 24028.09 & GALAXY & $0.094013 \pm 0.000005$ \\
\hline 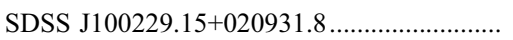 & 100229.15 & 20931.82 & QUASAR & $1.517140 \pm 0.000587$ \\
\hline SDSS J100231.43+023942.7 …......................... & 100231.43 & 23942.76 & GALAXY & $0.505580 \pm 0.000011$ \\
\hline 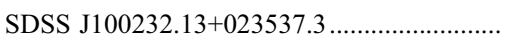 & 100232.13 & 23537.32 & QUASAR & $0.657166 \pm 0.000088^{b}$ \\
\hline SDSS J100232.29+022939.5 & 100232.29 & 22939.58 & STAR & \\
\hline 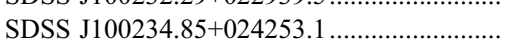 & 100234.85 & 24253.17 & QUASAR & $0.195840 \pm 0.000086^{\mathrm{b}}$ \\
\hline SDSS J100241.83+022525.3 …………............ & 100241.83 & 22525.35 & GALAXY & $0.359499 \pm 0.000018$ \\
\hline SDSS J100241.88+023314.0 & 100241.88 & 23314.04 & GALAXY & $0.186241 \pm 0.000011$ \\
\hline SDSS J100244.65+021152.8 & 100244.65 & 21152.83 & STAR & $\ldots$ \\
\hline SDSS J100246.33+021917.0 & 100246.33 & 21917.04 & GALAXY & $0.178724 \pm 0.000060$ \\
\hline SDSS J100246.50+024549.4 & 100246.50 & 24549.46 & GALAXY & $0.881450 \pm 0.000033$ \\
\hline SDSS J100249.33+023746.5 ………….............. & 100249.33 & 23746.52 & QUASAR & $2.131780 \pm 0.000603$ \\
\hline 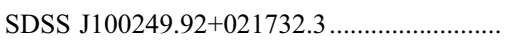 & 100249.92 & 21732.31 & QUASAR & $1.092280 \pm 0.001263$ \\
\hline 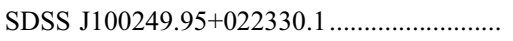 & 100249.95 & 22330.19 & GALAXY & $0.485346 \pm 0.000017$ \\
\hline SDSS J100250.20+023849.0 & 100250.20 & 23849.05 & GALAXY & $0.094168 \pm 0.000012$ \\
\hline 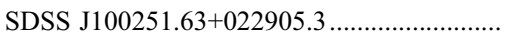 & 100251.63 & 22905.38 & QUASAR & $2.006750 \pm 0.000215^{\mathrm{b}}$ \\
\hline SDSS J100254.93+023515.6 $\ldots \ldots \ldots \ldots \ldots \ldots \ldots \ldots$ & 100254.93 & 23515.61 & STAR & \\
\hline 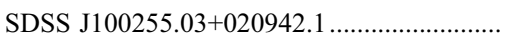 & 100255.03 & 20942.12 & GALAXY & $0.325148 \pm 0.000020$ \\
\hline 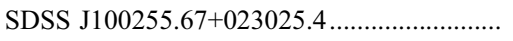 & 100255.67 & 23025.41 & GALAXY & $0.268544 \pm 0.000007$ \\
\hline SDSS J100255.69+023616.0 & 100255.69 & 23616.02 & GALAXY & $0.375908 \pm 0.000015$ \\
\hline 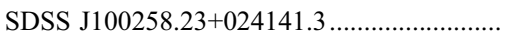 & 100258.23 & 24141.38 & GALAXY & $0.199750 \pm 0.000024$ \\
\hline 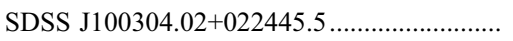 & 100304.02 & 22445.54 & GALAXY & $0.371347 \pm 0.000015$ \\
\hline 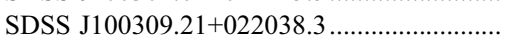 & 100309.21 & 22038.32 & QUASAR & $1.967030 \pm 0.000762^{\mathrm{b}}$ \\
\hline 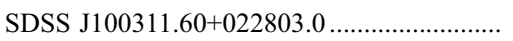 & 100311.60 & 22803.00 & GALAXY & $0.366929 \pm 0.000033$ \\
\hline SDSS J100315.99+022615.0 & 100315.99 & 22615.00 & QUASAR & $2.031070 \pm 0.000367$ \\
\hline
\end{tabular}

Notes.-Units of right ascension are hours, minutes, and seconds, and units of declination are degrees, arcminutes, and arcseconds. Table 3 is also available in machine-readable form in the electronic edition of the Astrophysical Journal.

${ }^{a}$ Redshift errors are formal errors returned by $\chi^{2}$ template-fitting procedure and may be underestimates in the case of systematic shifts according to line species (Richards et al. 2002a; McIntosh et al. 1999; Tytler \& Fan 1992).

${ }^{\mathrm{b}}$ Independently confirmed by the $2 \mathrm{dF}$ quasar survey or subsequent SDSS spectroscopic follow-up.

${ }^{c}$ BAL quasar with uncertain redshift.

d Redshift determined using a flux-weighted mean line centroid. Redshift errors are $\Delta z \sim 0.002$ or less. 


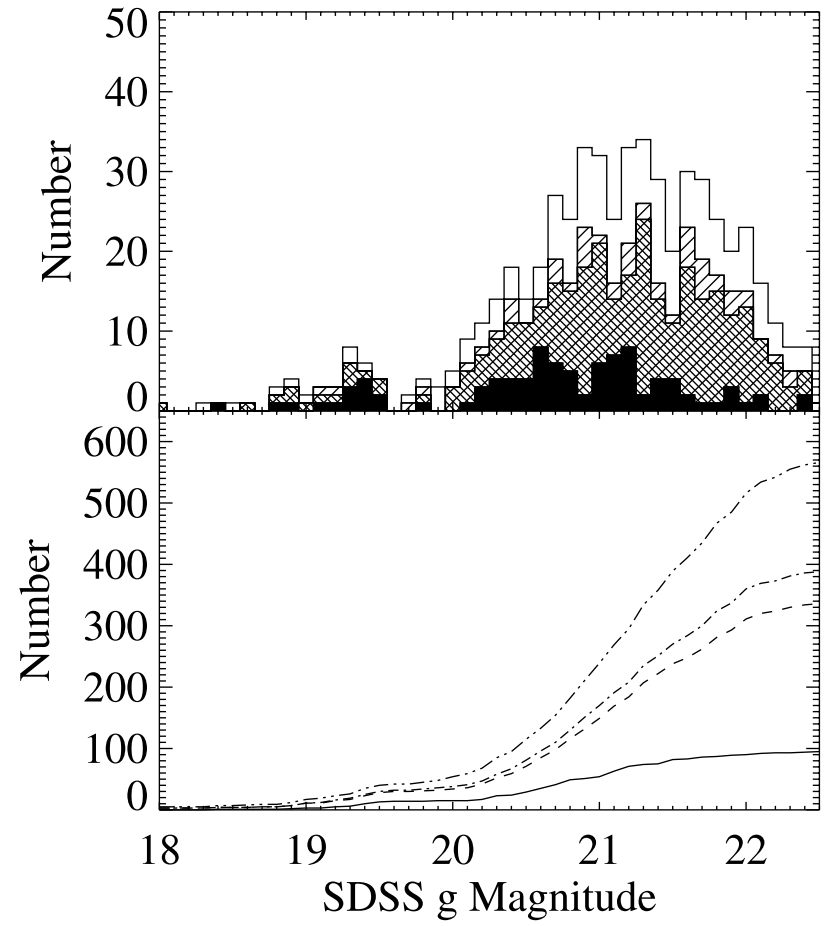

FIG. 4.-Top: Histogram of SDSS quasar yield, in 0.1 mag bins. The empty layer contains all SDSS targets in the $2 \mathrm{deg}^{2}$ COSMOS field; the wide-hatched layer, all SDSS targets within the pointing; the narrow-hatched layer, all SDSS targets observed in this study; and the solid layer, all SDSS targets confirmed in this study to be quasars. The plot excludes five bright candidate outliers; three of the five were targeted, but none were confirmed to be quasars. Bottom: Same layers plotted as a cumulative histogram.

fraction of $\sim 0.27$ translates into a prediction that $\sim 50$ of our 184 confirmed galaxies are in fact type 2 objects.

\section{DISCUSSION}

\subsection{Optimizing Quasar Selection}

The SDSS quasar flags based on finding outliers from the stellar locus in four-color space allowed us to select and confirm 95 quasars in the COSMOS field. At brighter magnitudes

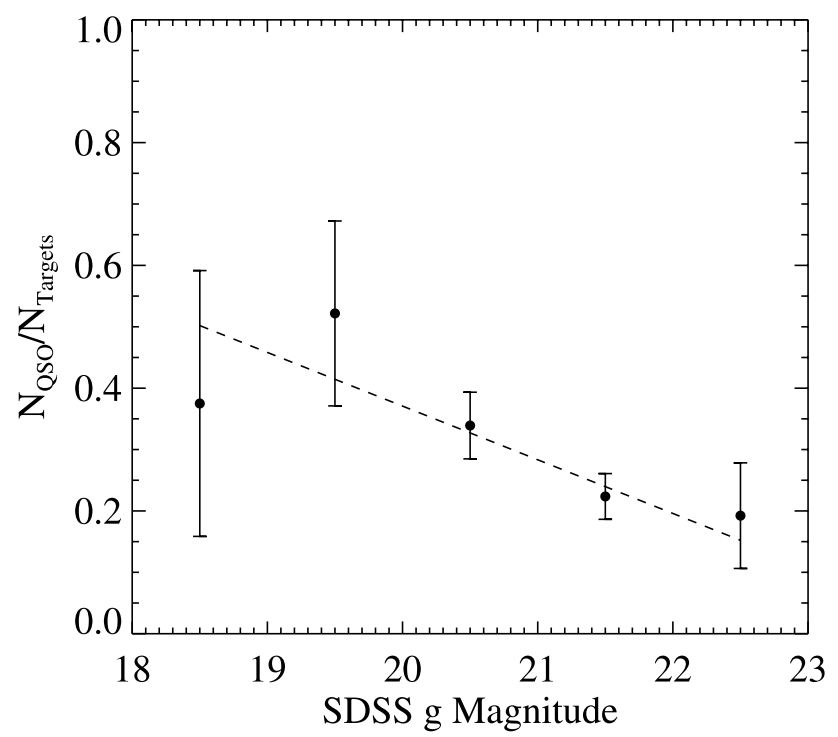

FIG. 5.- Success rate of confirming quasars per $1.0 \mathrm{mag}$ bin and the linear fit used to predict yields from the full target sample.
TABLE 4

Quasar Surface Density Projections

\begin{tabular}{ccc}
\hline \hline Parameter & Observed Field & COSMOS Field \\
\hline Number of quasars (this study) .............. & $110-127$ & $159-184$ \\
Number of additional SDSS quasars........ & 25 & 44 \\
Total area $\left(\mathrm{deg}^{2}\right) \ldots \ldots \ldots \ldots \ldots \ldots \ldots \ldots \ldots \ldots \ldots \ldots \ldots . . \ldots \ldots \ldots \ldots \ldots \ldots \ldots$ & 1.39 & 2.00 \\
Surface density at $g<22.5\left(\mathrm{deg}^{-2}\right) \ldots \ldots \ldots$. & $97-109$ & $102-114$ \\
\hline
\end{tabular}

$(g<19)$, the success rate was around $50 \%$, similar to our expectation from previous work using ultraviolet excess (UVX) quasar candidate selection and from the SDSS estimates. McIntosh et al. (2004) used a slightly smaller sample of quasars to derive an overall UVX selection efficiency of $61 \%$ down to $B \sim 22$. Richards et al. (2002b) find an overall efficiency better than $65 \%$ for the SDSS quasar target selection algorithm down to their limiting magnitude for spectroscopic follow-up of $i^{*}=19.1$ for the low-redshift and $i^{*}=20.2$ for the high-redshift selection. At dimmer magnitudes $(g>19)$, however, our fraction of confirmed quasars dropped steadily to below $15 \%$, in step with a rise in the primary contaminant population, emission-line galaxies. The fraction of stars and unconfirmed objects also increased to dimmer magnitudes, particularly in the last magnitude bin $21.5>g>$ 22.5. Figure 8 demonstrates these trends graphically.

Figure 9 shows the various subsamples in color-color space. As seen in Figure 3, the previously confirmed SDSS quasars lie

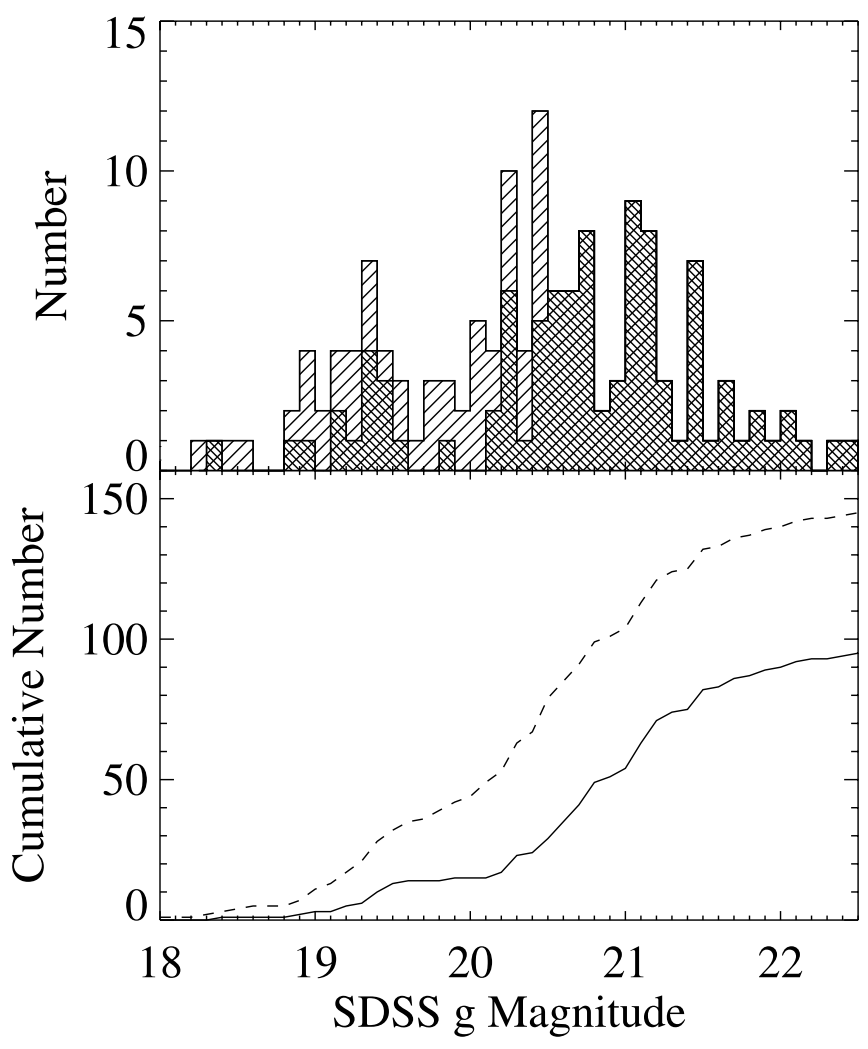

FIG. 6.-Top: Histogram of confirmed quasars in the COSMOS field. The narrow-hatched histogram indicates quasars confirmed only in this study. The wide-hatched histogram is a sum of all quasars confirmed in this field, both previously as part of SDSS spectroscopic follow-up and in the current work, taking into account sample overlaps. Bottom: Cumulative histogram of confirmed quasars within the $2 \mathrm{deg}^{2}$ COSMOS field. Quasars from this study alone are shown in the solid line, while the dashed line includes confirmed quasars from both SDSS spectroscopic follow-up and the current work. 


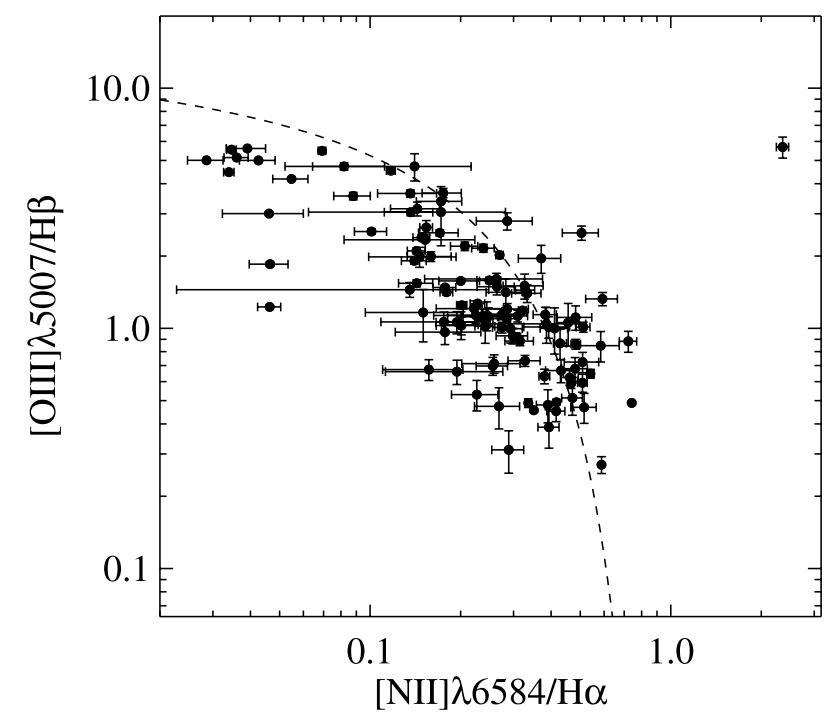

FIG. 7.-BPT (Baldwin et al. 1981) diagram for our sample of narrow-line objects below a redshift of 0.38 ; error bars are defined as the square root of the typical line width in pixels multiplied by the rms scatter per pixel measured from the continuum near the line. The dashed line is the demarcation between starforming galaxies and AGNs defined by Kauffmann et al. (2003), with starforming galaxies to the lower left and AGNs to the right on this plot.

at bluer $g-r$ and $u-g$ colors. Quasars discovered in the current work, along with the small number of stars, lie in the same region of color-color space as the previously confirmed SDSS quasars, while the contaminant emission-line galaxy population corresponds to targets with substantially redder $g-r$ colors. This work suggests that when taken to fainter magnitudes, the SDSS quasar flags select an increasingly large number of emission-line galaxies. Why do so many emission-line galaxies make it into the SDSS-selected sample? The primary reason is that the selection algorithm retains objects with $g-r$ colors of a low-redshift quasar but redder than the typical quasar in our sample. In the top left panel of Figure 10 we plot the $g-r$ histograms for the SDSS confirmed quasars and galaxies. There is a clear offset in mean color

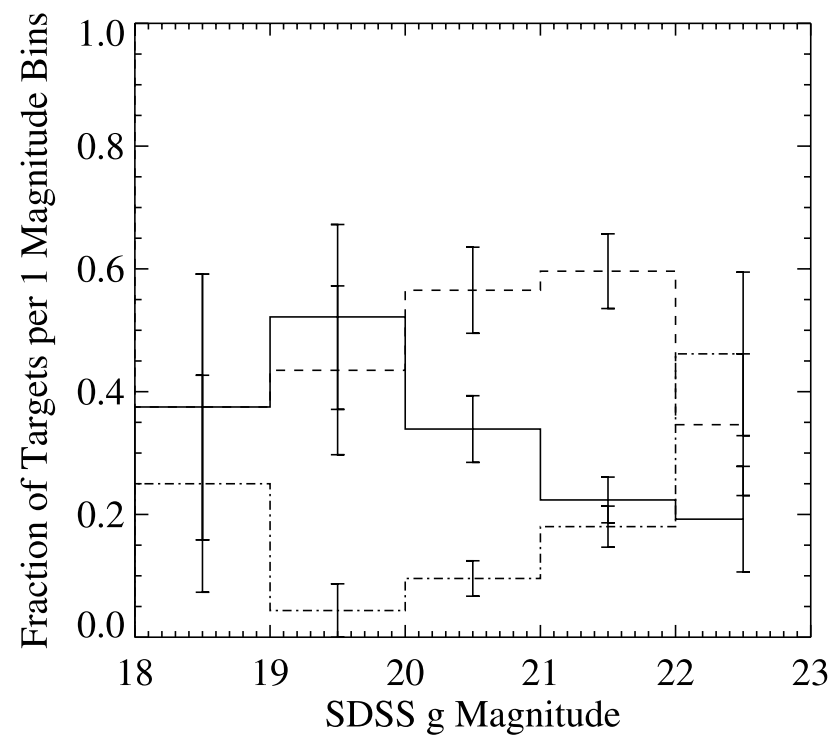

FIG. 8.-Fraction of SDSS targets per 1 mag bin that are quasars (solid line), galaxies (dashed line), or stars/unconfirmed (dot-dashed line). between the two samples: 0.18 for the quasars and 0.67 for the galaxies.

Fan (1999) performed simulations of the colors and sizes of compact emission-line galaxies (CELGs) to predict how much they would contaminate the target pool produced by the SDSS quasar target selection algorithm. He found that the colors of CELGs are dominated by the continuum due to the large bandwidth of the SDSS filters, noting that an emission-line equivalent width (EW) of $100 \AA$ will alter the galaxy magnitude in a given filter by only $0.1 \mathrm{mag}$. Galaxies with extremely large EWs indeed have peculiar colors, but they will show up in regions of color space far from quasars and normal stars. Furthermore, he found that the CELG population will be redder on average than the quasars, due to the redder continuum, but in his simulations this effect is critically dependent on the choice of power-law distribution. Hall et al. (1996) suggested that a limit of $B-V<$ 0.6 (or $g-r<0.4$ using a Fukugita et al. [1996] calibration) would eliminate half of the CELG population from the candidate pool. Fan (1999) concluded similarly that a cut of $g-r<0.35$ would eliminate about $60 \%$ of CELGs while retaining the largest number of quasars. This cut is shown for reference in all four panels of Figure 10.

We can now ask the same question of our data set: what $g-r$ cut would have maximized quasar selection efficiency in our sample? The top right panel of Figure 10 shows the number of quasars and galaxies retained in the sample for a particular $g-r$ cut; the cut retains all objects with $g-r$ less than the given value. The number of quasars rises first for lower cut values but is quickly surpassed by the galaxy counts beyond values of $g-$ $r=0.6$. The bottom left panel of Figure 10 gives the fraction of quasars, galaxies, and stars/unconfirmed objects for the same range of $g-r$ cuts. There is an obvious and broad peak in the quasar fraction for cuts in the range $0.0<g-r<0.4$. However, the ideal cut should maximize both the efficiency of the selection as well as the total number of quasars found. The bottom right panel of Figure 10 plots the fraction of quasars and galaxies versus the number of quasars retained if a given $g-r$ cut is applied. The point of maximum efficiency and maximum number of quasars occurs at a $g-r$ cut of $\sim 0.35$, in complete agreement with Fan (1999) and Hall et al. (1996). This color cut reduces the number of emission-line galaxies from 184 to 20 , i.e., it removes $89 \%$ of the CELGs, an even more drastic improvement than achieved by Hall et al. (1996) and Fan (1999). The cut also removes 16 quasars from our sample but increases the mean quasar fraction from $33 \%$ to $57 \%$, a level in better agreement with previous UVX-selected studies done at brighter magnitudes. Even higher efficiency has been demonstrated by Richards et al. (2004) with the SDSS DR1 photometric data by applying a probability density analysis to training sets and using a nonparametric Bayesian classification. They achieve an efficiency of $\sim 95 \%$ down to $g=21$ while maintaining a completeness of $94.7 \%$ to unresolved quasars brighter than $g \sim 19.5$. At fainter magnitudes, however, Richards et al. (2004) expect reduced completeness.

It should be noted that when working with state-of-the-art multiobject spectrographs over relatively moderate sized fields, optimizing the detection efficiency of a particular category of target is becoming an unnecessary or even unwanted precaution. The large number of fibers per square degree on the sky can compensate to some extent for a decrease in selection efficiency without costing additional observing time. Furthermore, any simple cut will inevitably reduce the completeness of the survey; as mentioned above, the cut of $g-r<0.35$ would have removed $16(17 \%)$ of the SDSS spectroscopically confirmed quasars. For 


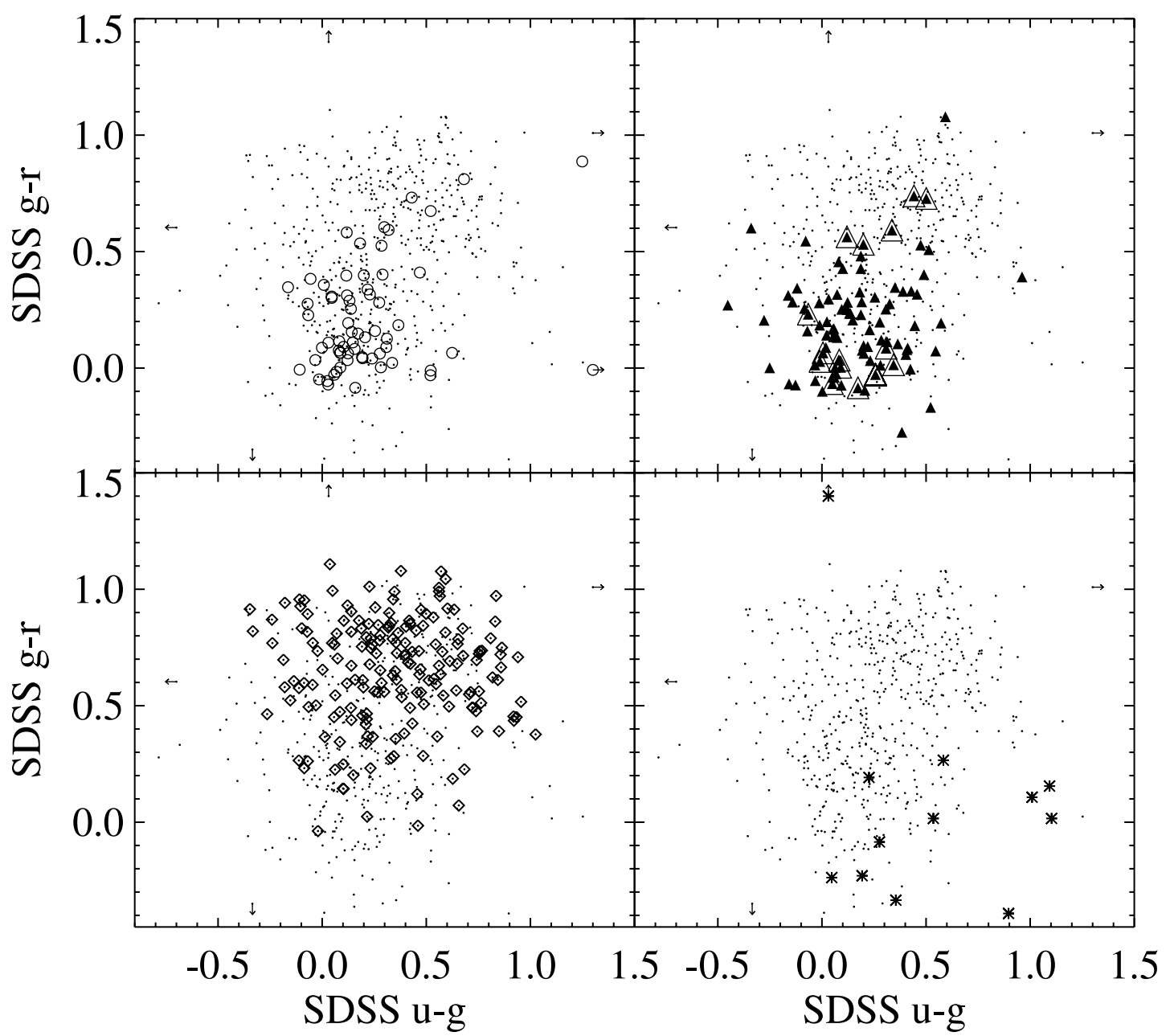

FIG. 9.-Color-color plots of SDSS $g-r$ vs. $u-g$. Top left: Quasar candidates in this study are shown in small circles and previously confirmed SDSS quasars in large circles. Top right: Quasar candidates in this study are small circles, and those confirmed are filled triangles. Overlapping quasars between the SDSS spectroscopic follow-up and this study are shown as large triangles. Improvements in photometry between DR1 and DR4 for the previously confirmed quasars offset points slightly between the top two panels. Bottom left: Quasar candidates in this study are small circles, and those confirmed as galaxies are diamonds. Bottom right: Quasar candidates in this study are small circles, and those confirmed as stars are denoted with asterisks. 

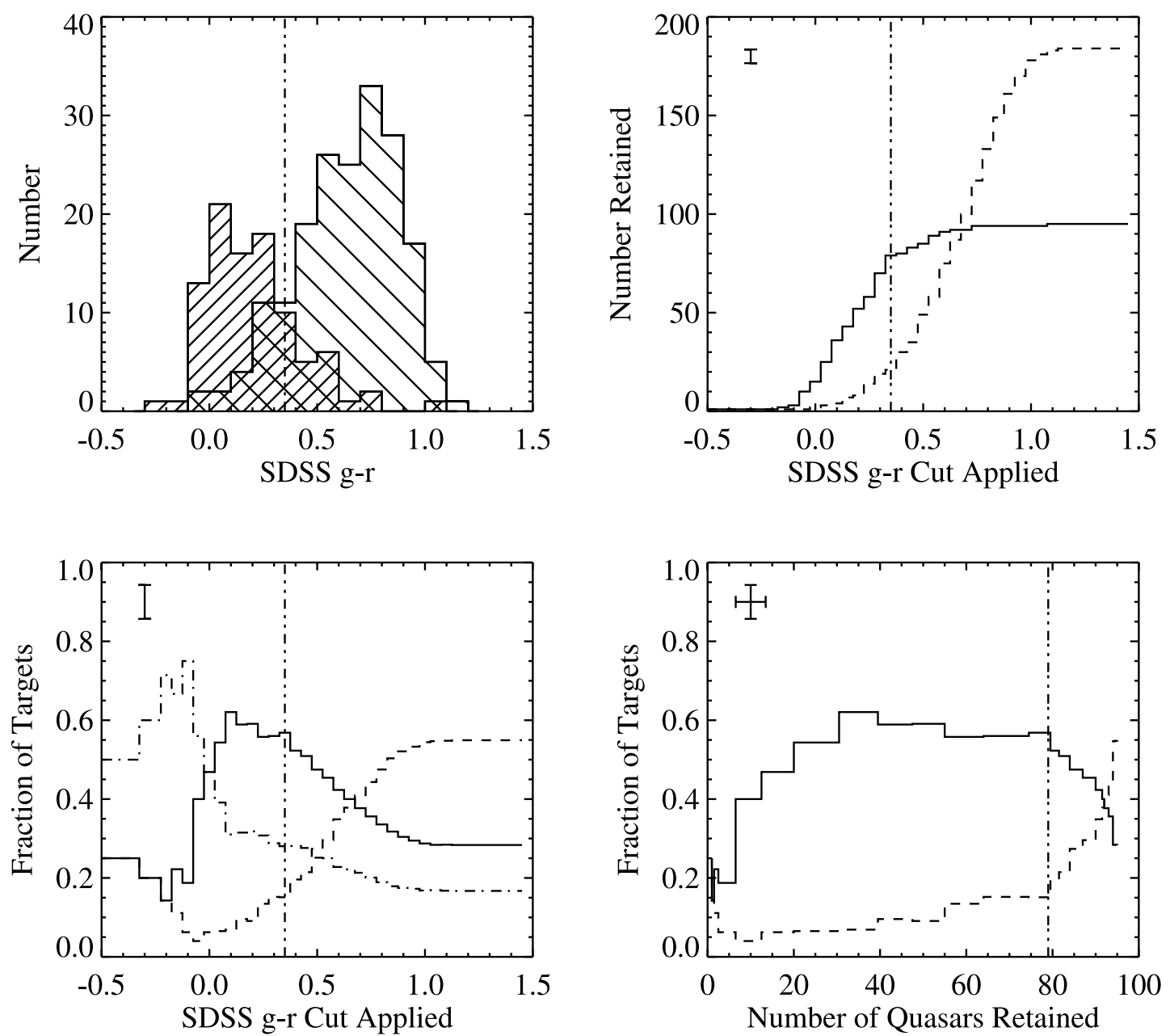

FIG. 10.-Top left: SDSS confirmed quasar and galaxy $g-r$ histograms. Quasars are shown as a narrow-hatched histogram, and galaxies as a wide-hatched one. The vertical line at $g-r=0.35$ is the cut discussed in the text and is replicated on all panels. In the remaining panels quasars are shown as a solid line, galaxies as a dashed line, and stars/unconfirmed objects as a dot-dashed line. The mean error bars for the quasars are shown in the upper left-hand corner. Top right: Number of SDSS confirmed quasars and galaxies retained in the final sample vs. the $g-r$ cut applied. The cut retains all objects with $g-r$ less than the given value. Bottom left: Fraction of SDSS confirmed quasars, galaxies, and stars/unconfirmed objects that would be retained in the sample vs. the $g-r$ cut applied. Bottom right: Fraction of SDSS confirmed quasars and galaxies retained in the sample vs. the number of quasars retained if a $g-r$ cut is applied.

the current study, the goal is to find as many quasars as possible within one field in order to have a dense grid of absorption-line probes of the intervening matter; thus, completeness is more important than efficiency.

\subsection{Emission-Line Galaxies}

The redshift distribution of our sample is shown in Figure 11. The emission-line galaxies have a median redshift of 0.3 , while the median redshift of the quasars is 1.4. The redshift range of our sample of emission-line galaxies is 0.006-0.9; the quasar sample spans redshifts from 0.2 to nearly 2.3 . The top panel of Figure 12 shows $g-r$ color versus redshift for the confirmed quasars and galaxies. While the quasars show a steady falloff toward higher redshift, the emission-line galaxies peak in $g-r$ color around redshifts of $0.1-0.6$. Again, it is this red, lowredshift population of CELGs that is the primary contaminant in this work. The lower panel of Figure 12 gives the SDSS $g$-band luminosity $\left(\nu L_{\nu}\right)$ in ergs $\mathrm{s}^{-1}$ versus redshift for both the quasar and galaxy samples. There is a separation between the quasars and galaxies, with quasars filling out the higher luminosity end and the galaxies residing at lower luminosities. For reference, tracks of the redshift evolution of $L_{*}$ in the rest-frame $g, u, 2800 \AA$, and $1500 \AA$ bands are shown taken from work on the FORS (Focal

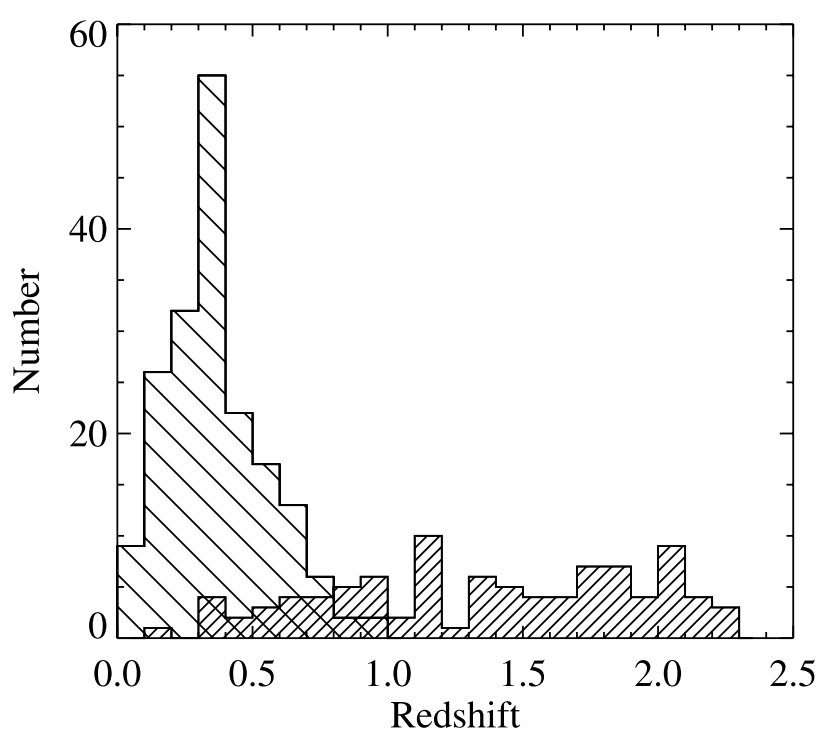

FIG. 11.-SDSS confirmed quasar and galaxy redshift histograms. Quasars are shown as a narrow-hatched histogram, and galaxies as a wide-hatched one. 


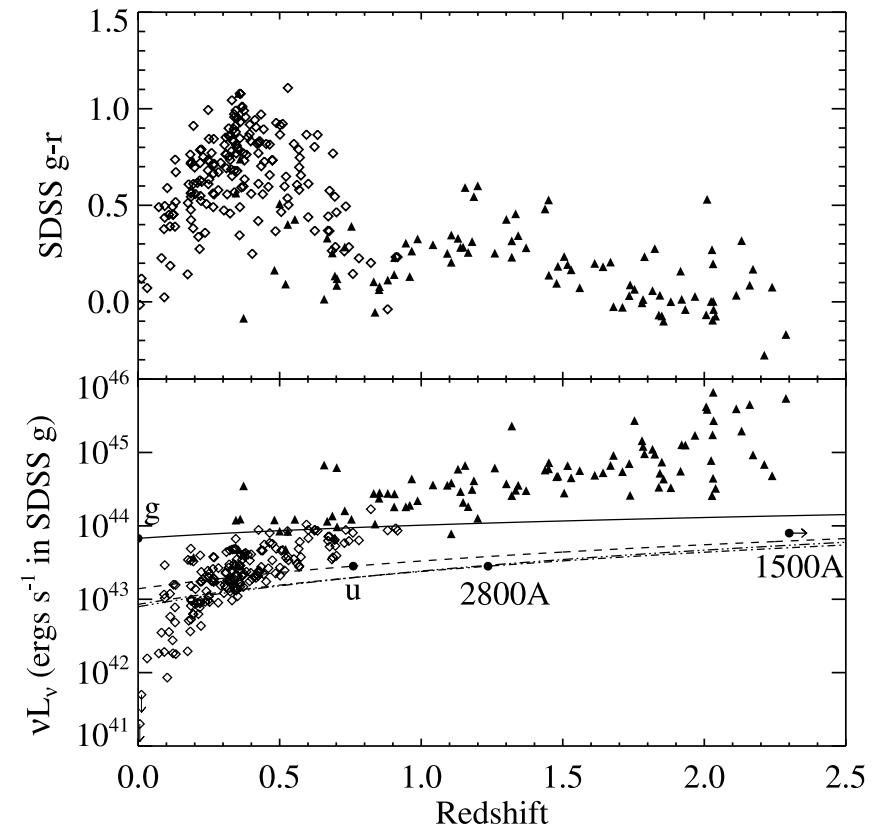

FIG. 12.-Top: SDSS $g-r$ vs. redshift for the confirmed quasar ( filled triangles) and galaxy (diamonds) samples in this study. Bottom: SDSS $g$ luminosity vs. redshift for the quasar and galaxy samples. The tracks show the evolution of an $L^{*}$ galaxy in rest-frame SDSS $g$ (solid line), $u$ (dashed line), $2800 \AA$ (dot-dashed line), and $1500 \AA$ (double-dot-dashed line) from the FORS Deep Field (Gabasch et al. 2004). The 2800 and $1500 \AA$ tracks are nearly indistinguishable. The filled circles represent the redshifts at which each restframe $L^{*}$ track passes through the observed $g$ band.

Reducer/Low Dispersion Spectrograph) Deep Field (Gabasch et al. 2004). The positions in redshift where these rest-frame bands pass through the observed $g$ band are shown as filled circles. Thus, when observed in the $g$ band, an $L^{*}$ galaxy at $z \sim 0.75$ would correspond in luminosity to the point labeled " $u$." The galaxy population straddles $L^{*}$ at a redshift of $\sim 0.4$.

\subsection{BAL Fraction}

The final topic of discussion is that of the fraction of broad absorption line (BAL) quasars in our sample. Our spectra are not well suited to a rigorous BAL selection algorithm involving continuum fitting such as those used by Tolea et al. (2002) and Reichard et al. (2003) because of the lack of true flux calibration, the moderate to low $\mathrm{S} / \mathrm{N}$ for the faint objects, and the cosmetic issues described in $\S 3$. However, from a visual inspection we would expect to be able to identify the most extreme cases, namely, the LoBAL or LoBAL + HiBAL cases. LoBAL quasars show broad absorption troughs associated with low-ionization species such as $\mathrm{Mg}$ II as well as higher absorption species such as $\mathrm{C}$ IV and $\mathrm{C}$ III. Conversely, HiBAL quasars show broad absorption of high-ionization species only, while the LoBAL + HiBAL designates an intermediate category. Reichard et al. (2003) present composite spectra from SDSS of each of these classifications. From a visual inspection of the SDSS confirmed quasars in the COSMOS field, we find that two are likely LoBAL quasars, while none of the previously confirmed quasars stand out as obvious LoBAL quasars. This corresponds to an observed LoBAL fraction of $1.7 \%$ for our SDSS sample.

Due to the small sample, we cannot derive the appropriate correction for selection effects; however, we note that Reichard et al. (2003) find no sizable correction factor for BAL quasars in the redshift range of our study $(z<2.3)$. We can therefore compare our rough LoBAL fraction with that of other studies; Menou et al. (2001) find a corrected LoBAL fraction of $2.8 \% \pm 1.1 \%$ or a more conservative estimate of $2.0 \% \pm 0.9 \%$ using early SDSS data matched to the FIRST radio survey catalog. Reichard et al. (2003) find a similar LoBAL fraction of $1.9_{-0.4}^{+0.5} \%$ in the SDSS Early Data Release. Thus, our sample of quasars in the COSMOS field appears to be consistent with the LoBAL fractions quoted in previous SDSS studies. Assuming a typical LoBAL to HiBAL distribution in the COSMOS field, we extrapolate that the results of a more thorough analysis of the overall BAL fraction would also be in agreement with these previous studies.

\section{CONCLUSIONS}

In this study we used the MMT and the Hectospec instrument to confirm a sample of 95 quasars in the $2 \mathrm{deg}^{2}$ COSMOS field. These quasar candidates were selected from the SDSS DR 1 catalog using flags assigned by the SDSS multicolor quasar target selection algorithm. Our findings are consistent with quasar surface densities of $102 \pm 7 \mathrm{deg}^{-2}$ over the entire COSMOS field down to $g=22.5$. From the current work, we find that the quasar fraction using the SDSS selection algorithm is $50 \%$ for $g<20$, falling to below $15 \%$ at our magnitude limit of $g=22.5$. Of the 95 confirmed quasars, at least two are BAL quasars. The primary contaminants to the sample are 184 emission-line galaxies with $g-r$ colors greater than 0.35 and redshifts between 0.2 and 0.6 . If efficiency is crucial in a color-selected survey, a cut retaining only objects with $g-r<0.35$ can be applied, removing over $80 \%$ of these compact emission-line galaxies and increasing the quasar yield to $\sim 60 \%$ for magnitudes as faint as $g=22.5$.

With a confirmed quasar population of 139, the COSMOS field is now one of the most densely sampled regions where a grid of sight lines can be used to identify absorbers as probes of a large contiguous volume. Ongoing work is extending the quasarconfirmation phase of this project down to $g \sim 23.5$ (Trump et al. 2006; Impey et al. 2006). Once a sample of quasar candidates exists for the entire field, the second phase of the project will involve using higher resolution spectra of these quasar probes to detect absorbers along the line of sight. These absorber data, which trace diffuse IGM and galaxy halos, will be combined with the COSMOS dark matter and galaxy redshift surveys in order to study the three-dimensional distribution of dim and luminous matter in the COSMOS field.

We are grateful to Cathy Petry and Xiaohui Fan for advice during the project and to Kris Eriksen, Andy Marble, and Daniel Eisenstein for observing assistance. M. P. was supported by an NSF Graduate Research Fellowship. C. D. I. acknowledges support of this research through NASA award HST-GO-10092.04 to the University of Arizona.

Funding for the Sloan Digital Sky Survey (SDSS) has been provided by the Alfred P. Sloan Foundation, the Participating Institutions, the National Aeronautics and Space Administration, the National Science Foundation, the U.S. Department of Energy, the Japanese Monbukagakusho, and the Max Planck Society. The SDSS Web site is http://www.sdss.org.

The SDSS is managed by the Astrophysical Research Consortium (ARC) for the Participating Institutions. The Participating Institutions are The University of Chicago, Fermilab, the Institute for Advanced Study, the Japan Participation Group, The Johns Hopkins University, Los Alamos National Laboratory, 
the Max-Planck-Institute for Astronomy (MPIA), the Max-PlanckInstitute for Astrophysics (MPA), New Mexico State University, University of Pittsburgh, Princeton University, the United States Naval Observatory, and the University of Washington.
This research has made use of the NASA/IPAC Extragalactic Database (NED), which is operated by the Jet Propulsion Laboratory, California Institute of Technology, under contract with the National Aeronautics and Space Administration.

\section{APPENDIX}

\section{DISCOVERY SPECTRA}

Figure Set 13 presents our quasar and galaxy discovery spectra, given in order of right ascension. Each panel is labeled with the object name, classification, and redshift.

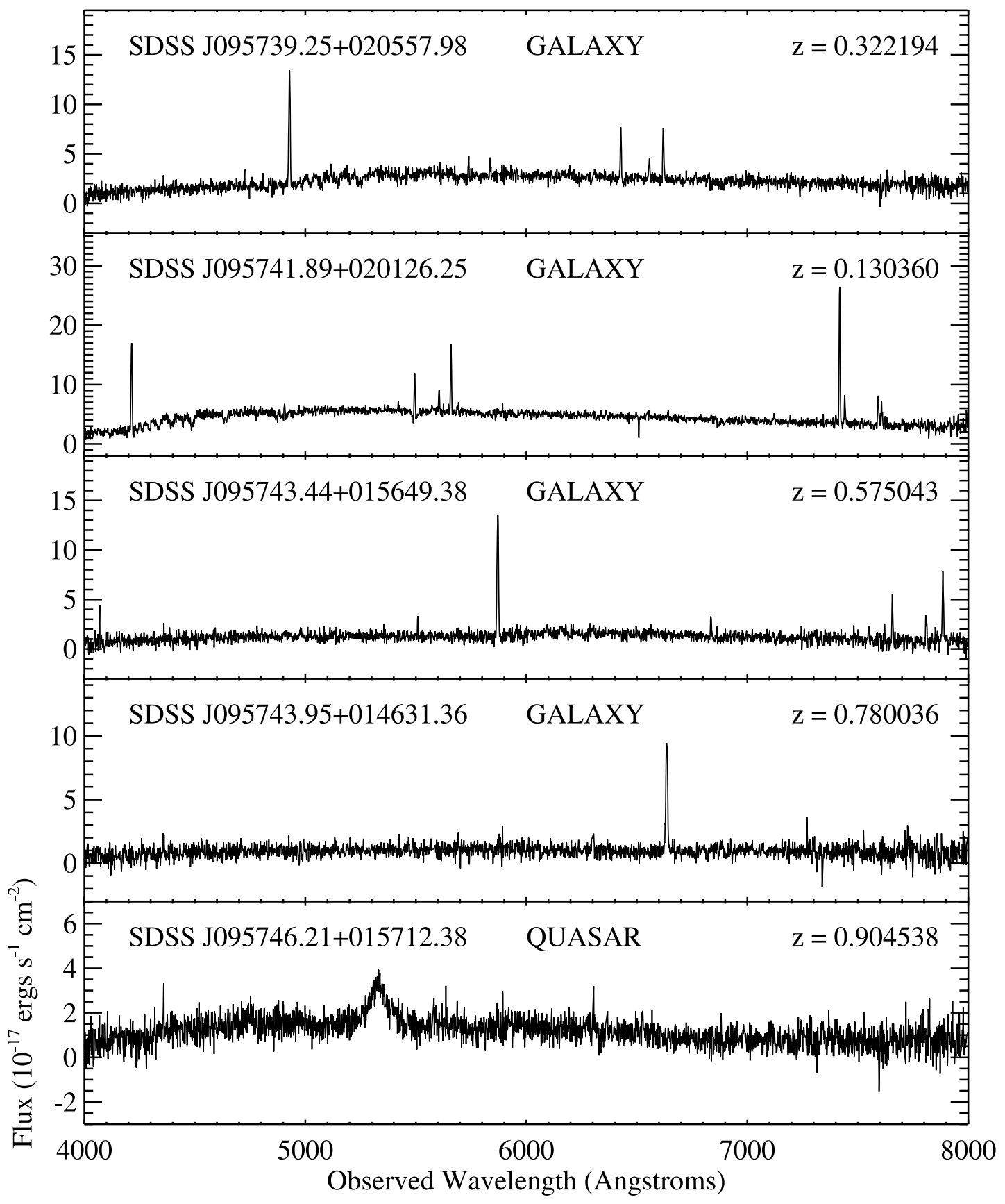

FIG. 13.1. SDSS J095739.25+020557.98-SDSS J095746.21+015712.38

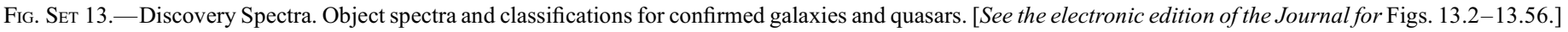


Abazajian, K., et al. 2003, AJ, 126, 2081

Adelman-McCarthy, J. K., et al. 2006, ApJS, 162, 38

Bahcall, J. N., et al. 1993, ApJS, 87, 1

Baldwin, J. A., Phillips, M. M., \& Terlevich, R. 1981, PASP, 93, 5

Boyle, B. J., Shanks, T., Croom, S. M., Smith, R. J., Miller, L., Loaring, N., \& Heymans, C. 2000, MNRAS, 317, 1014

Cohen, J. G., Cowie, L. L., Hogg, D. W., Songaila, A., Blandford, R., Hu, E. M., \& Shopbell, P. 1996, ApJ, 471, L5

Cohen, J. G., Hogg, D. W., Pahre, M. A., Blandford, R., Shopbell, P. L., \& Richberg, K. 1999, ApJS, 120, 171

Croom, S. M., Smith, R. J., Boyle, B. J., Shanks, T., Miller, L., Outram, P. J., \& Loaring, N. S. 2004, MNRAS, 349, 1397

Dinshaw, N., \& Impey, C. D. 1996, ApJ, 458, 73

Fabricant, D., et al. 2004, Proc. SPIE, 5492, 767

Fan, X. 1999, AJ, 117, 2528

Fukugita, M., Ichikawa, T., Gunn, J. E., Doi, M., Shimasaku, K., \& Schneider, D. P. 1996, AJ, 111, 1748

Gabasch, A., et al. 2004, A\&A, 421, 41

Grogin, N. A., \& Geller, M. J. 1998, ApJ, 505, 506

Hall, P. B., Osmer, P. S., Green, R. F., Porter, A. C., \& Warren, S. J. 1996, ApJ, 471, 1073

Heisler, J., Hogan, C. J., \& White, S. D. M. 1989, ApJ, 347, 52

Impey, C. D., et al. 2006, ApJ, submitted

Kauffmann, G., et al. 2003, MNRAS, 346, 1055

Liske, J., Webb, J. K., Williger, G. M., Fernández-Soto, A., \& Carswell, R. F. 2000, MNRAS, 311, 657
REFERENCES

Loh, J.-M., Quashnock, J. M., \& Stein, M. L. 2001, ApJ, 560, 606

Menou, K., et al. 2001, ApJ, 561, 645

Meylan, G., ed. 1995, QSO Absorption Lines (Berlin: Springer)

McIntosh, D. H., Impey, C. D., \& Petry, C. E. 2004, AJ, 128, 544

McIntosh, D. H., Rix, H.-W., Rieke, M. J., \& Foltz, C. B. 1999, ApJ, 517, L73

Penton, S. V., et al. 2002, ApJ, 565, 720

Reichard, T. A., et al. 2003, AJ, 126, 2594

Richards, G. T., Vanden Berk, D. E., Reichard, T. A., Hall, P. B., Schneider,

D. P., SubbaRao, M., Thakar, A. R., \& York, D. G. 2002a, AJ, 124, 1

Richards, G. T., et al. 2002b, AJ, 123, 2945 . 2004, ApJS, 155, 257 2005, MNRAS, 360, 839

Sargent, W. L. W., \& Steidel, C. C. 1987, ApJ, 322, 142

Schlegel, D. J., Finkbeiner, D. P., \& Davis, M. 1998, ApJ, 500, 525

Scoville, N. Z., et al. 2006, ApJ, submitted

Tolea, A., Krolik, J. H., \& Tsvetanov, Z. 2002, ApJ, 578, L31

Trump, J., et al. 2006, ApJ, submitted

Tytler, D., \& Fan, X.-M. 1992, ApJS, 79, 1

Vanden Berk, D. E., et al. 2005, AJ, 129, 2047

Weymann, R. J., et al. 1998, ApJ, 506, 1

Williger, G. M., Hazard, C., Baldwin, J. A., \& McMahon, R. G. 1996, ApJS, 104,145

Williger, G. M., Smette, A., Hazard, C., Baldwin, J. A., \& McMahon, R. G. 2000, ApJ, 532, 77

York, D. G., et al. 2000, AJ, 120, 1579 